\title{
1 Bidirectional modulation of pain-related behaviors in the zona incerta
}

2 Abbreviated title: Contribution of $\mathrm{ZI}$ to persistent pain

3

4 Sudhuman Singh ${ }^{1}$, Spring Valdivia ${ }^{1}$, Omar Soler-Cedeño ${ }^{1}$, Anisha P. Adke ${ }^{1}$, Barbara Benowitz ${ }^{1}$,

5 Daniela Velasquez ${ }^{1}$, Torri D. Wilson ${ }^{1}$, Yarimar Carrasquillo ${ }^{1 \#}$

6

$7 \quad{ }^{1}$ National Center for Complementary and Integrative Health, National Institute of Health,

8 Bethesda, MD, United States

9

10 \#Correspondence

11 Yarimar Carrasquillo, $\mathrm{PhD}$

12 National Center for Complementary and Integrative Health

13 National Institutes of Health

1435 Convent Drive

15 Building 35A / Room 1E-410

16 Bethesda, MD 20892

17 Phone: 301-451-8147

18 Fax: 301-480-0772

19 Email: yarimar.carrasquillo@nih.gov

20 


\section{Abstract}

22 Central amygdala neurons expressing protein kinase C-delta (CeA-PKCס) are sensitized

23 following nerve injury and promote pain-related responses in mice. The neural circuits

24 underlying modulation of pain-related behaviors by CeA-PKCס neurons, however, remain

25 unknown. In this study, we identified a functional monosynaptic inhibitory neural circuit

26 that originates in CeA-PKCס neurons and terminates in the ventral region of the zona

27 incerta $(\mathrm{ZI})$, a subthalamic structure previously linked to pain processing. Behavioral

28 experiments further show that chemogenetic inhibition of GABAergic ZI neurons is

29 sufficient to induce bilateral hypersensitivity in uninjured mice as well as contralateral

30 hypersensitivity after nerve injury. In contrast, chemogenetic activation of GABAergic ZI

31 neurons reverses nerve injury-induced hypersensitivity, demonstrating that silencing of

32 the $\mathrm{ZI}$ is required for injury-induced behavioral hypersensitivity. Our results identify a previously unrecognized inhibitory efferent pathway from CeA-PKCס neurons to the $\mathrm{ZI}$

34 and demonstrate that ZI-GABAergic neurons can bidirectionally modulate pain-related

35 behaviors in mice.

36 Keywords: pain, central amygdala, protein kinase $\mathrm{C}$ delta, zona incerta, GABAergic

37 neurons, amygdala circuit, pain pathways 


\section{Introduction}

Persistent pain resulting from lesions or diseases affecting the peripheral and central nervous system can severely affect a person's life over time if left untreated (Dworkin, 2002; Treede et al., 2008). Understanding the neural circuits underlying pain processing and how they are recruited in a maladaptive manner following injury is crucial for the development of improved treatment options for persistent pain. Several neuroimaging, pharmacological and electrophysiological studies in humans and animals

47 demonstrate that the amygdala is a key locus in persistent pain processing (Bernard and Besson, 1990; Zald, 2003; Neugebauer et. al., 2004; Carrasquillo and Gereau, 2007; Bushnell et. al., 2013). A recent study further demonstrated that the CeA can both enhance and decrease pain-related behaviors in a cell-type-specific manner (Wilson et al., 2019). CeA neurons expressing protein kinase C-delta (CeA-PKCס), for example, are sensitized by nerve injury and promote pain-related responses. In contrast, neurons expressing somatostatin are inhibited by nerve injury and promote decreases in pain-

54 related behaviors. The circuit and cellular mechanisms responsible for bidirectional modulation of pain-related responses in the CeA, however, are still unclear.

In the present study, we began to address this question by characterizing the

57 efferent projections from CeA-PKCס neurons. Our cell-type-specific anatomical experiments identified the zona incerta $(\mathrm{ZI})$ as one of the efferent targets of CeA-PKCס neurons. The $\mathrm{ZI}$ is a subthalamic nucleus located ventrolateral to the medial lemniscus and dorsomedial to the substantia nigra (Ricardo,1981). The $\mathrm{Zl}$ is comprised of heterogeneous groups of cells defined by the expression of molecular markers such as parvalbumin, tyrosine hydroxylase, somatostatin, calbindin, and glutamate (Mitrofanis, 
2005). The functions of the $\mathrm{Zl}$ are not fully understood but recent studies suggest that this brain region contributes to fear conditioning (Zhou et al., 2018) binge eating (Zhang and Van Den Pol, 2017), defensive behaviors (Chou et al., 2018) and predatory hunting (Zhao et al., 2019). In rodent models of pain, changes in neural activity have been reported in the Zl (Masri er al., 2009) and behavioral studies further show that experimentally modulating the activity of ZI neurons alters behavioral hypersensitivity (Petronilho et. al., 2012; Moon and Park, 2017; Hu et. al., 2019; Wang et. al., 2020). Most of the literature suggests, for example, that the $\mathrm{ZI}$ is inhibited in the context of pain and that inhibition drives behavioral hypersensitivity (Masri et. al., 2009; Moon et. al., 2016; Moon and Park, 2017; Hu et. al., 2019). A recent study, however, suggests the opposite- the $\mathrm{ZI}$ is activated in the context of pain and increases in neuronal activity drive hypersensitivity (Wang et. al., 2020). These seemingly conflicting results suggest that modulation of pain in the $\mathrm{Zl}$ is complex and, most likely, cell-type and circuit-specific. Identifying the sources of excitation and inhibition of $\mathrm{ZI}$ neurons in the context of pain will be important to begin untangling the mechanisms underlying modulation of pain in the Zl.

Based on our anatomical findings demonstrating a projection from CeA-PKC $\delta$ neurons to the $\mathrm{ZI}$, in combination with previous work showing that CeA-PKCס neurons are GABAergic and display increases in activity following injury, we hypothesized that inhibitory inputs from CeA-PKC $\delta$ neurons are a source of pain-related inhibition in the $\mathrm{ZI}$ that results in increases in pain-related behaviors. In the present study, we tested this hypothesis using a combination of cell-type-specific anatomical traces, opto-assisted circuit mapping, chemogenetic manipulations and behavioral assays to measure hypersensitivity in mice. Our combined results show that there is a functional 
86 monosynaptic inhibitory efferent pathway from CeA-PKCס neurons to the $\mathrm{ZI}$ and further

87 demonstrate that ZI-GABAergic neurons can bidirectionally modulate pain-related behaviors in mice.

\section{Results}

\section{Identification of CeA-PKCס neuronal efferent targets}

The neural pathways underlying modulation of pain-related behaviors by CeA-

PKCס neurons remains unknown. To begin to address this question, we stereotaxically

94 injected a cre-dependent adeno-associated virus (AAV) anterograde tracer (ChrimsonR

or mCherry) into the CeA of Prkcd-cre mice (Figure 1A). The transduction of ChrimsonR

or mCherry in CeA-PKC $\delta$ cells was confirmed with immunostaining for PKCठ (Figure 1B).

Mapping of ChrimsonR (or mCherry) positive axonal terminals revealed CeA-PKCठ

efferent projections with dense, moderate and sparse labeling in 17 brain regions

99 throughout the brain, including the basal forebrain, striatum, thalamus, hypothalamus,

our experiments have been previously defined as output regions of the CeA using approach to study the efferent projections of CeA-PKCס neurons. 
108 identified had either few or moderate numbers of terminals, with only three regions, 109 including the bed nucleus of stria terminalis, extended amygdala and parabrachial nucleus, containing high densities of labeling (Figure 1C-D and Table 1).

112 the bed nucleus of stria terminalis, extended amygdala and central amygdala of all 5 113 brains analyzed. Dense labeling was also seen in the lateral parabrachial nucleus of 3 of

114 the 5 brains analyzed, with sparse labeling observed in 1 brain and no labeling in the 115 other brain. Moderate to sparse labeling was consistently observed in the substantia 116 innominata in all 5 brains; in the zona incerta, para-subthalamic nucleus, substantia nigra 117 and reticular formation in 4 of 5 brains; and in the subthalamic nucleus and ventral 118 tegmental area in 3 of the 5 brains analyzed. Lastly, sparce labeling was consistently 119 seen in the globus pallidus, lateral preoptic area and lateral hypothalamus in all 5 brains; 120 in the locus coeruleus in 4 of 5 brains and in the pedunculopontine tegmental nucleus, 121 laterodorsal tegmental nucleus and periaqueductal grey in 3 of the 5 brains. Consistent 122 with previous studies using traditional anterograde tracers in the CeA (Shinonaga et al., 123 1992; Reardon and Mitrofanis, 2000; Barbier et al., 2017; Zhou et al., 2018; Aggleton, 124 2000), no terminal labeling was observed in cortical regions of any of the 5 brains 125 evaluated.

127 to the CeA (Figure 1 - figure supplement 1). The number of transduced neurons in 128 these mice were comparable to the expression levels of PKCठ-tdTomato neurons in the 129 CeA of Prkcd-cre::Ai9 mice, demonstrating robust transduction efficiency in these 
A

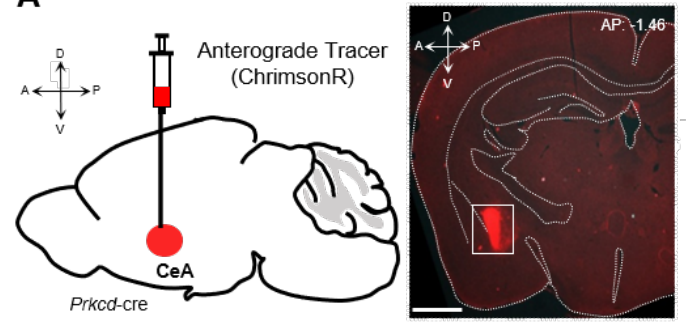

C

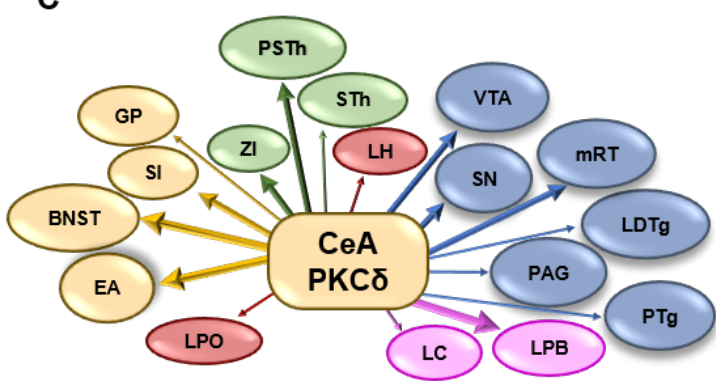

Hypothalamus

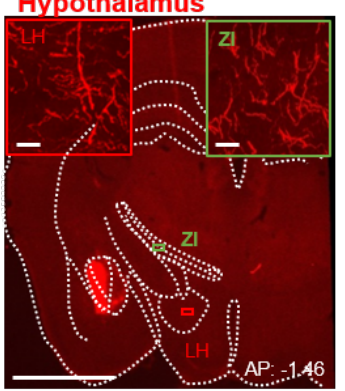

Midbrain

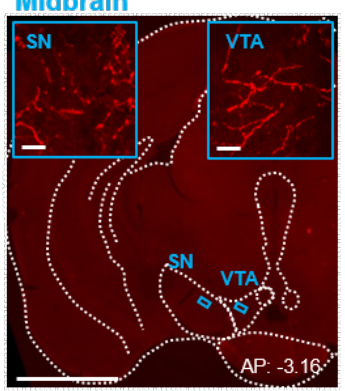

Thalamus

Pons
B
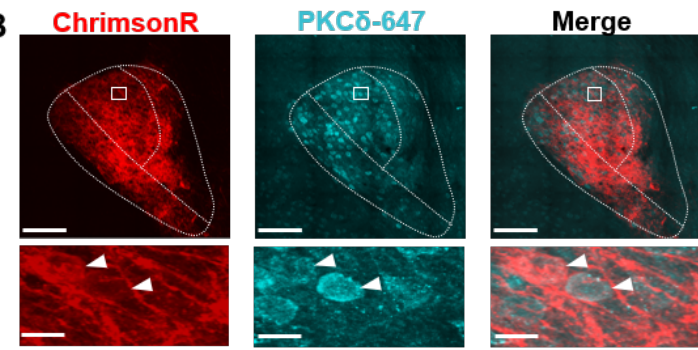

D Basal Forebrain
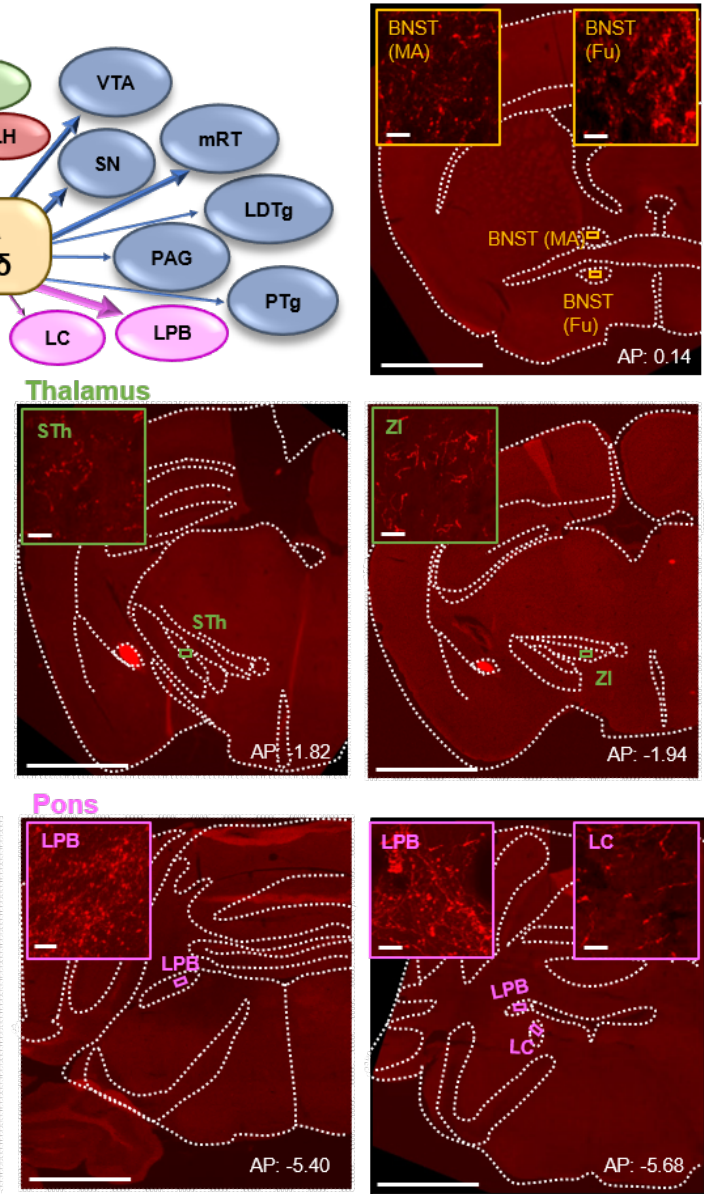

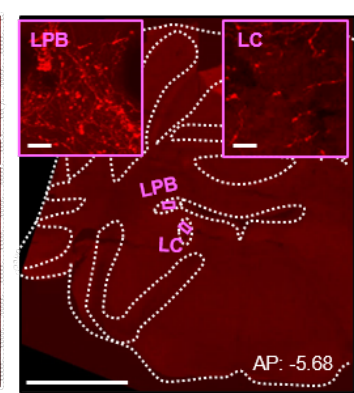

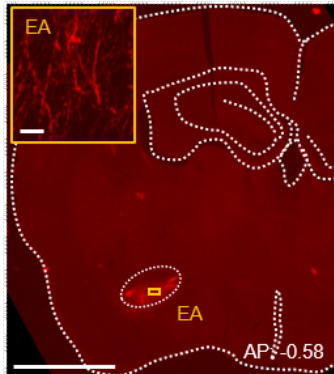

Midbrain

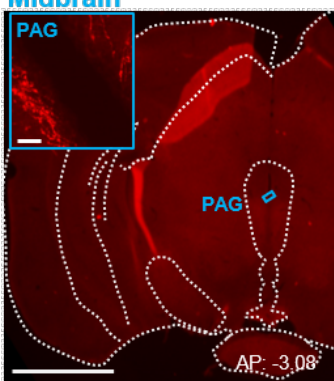

$E$

Proposed Model

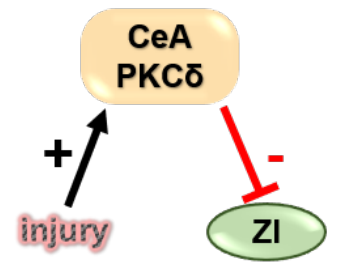

Figure 1. CeA-PKCס neurons efferent targets. (A) Schematic of experimental approach. ChrimsonR-tdTomato was unilaterally injected into the $\mathrm{CeA}$ of a $\mathrm{PKC} \delta$-cre mouse. A representative coronal brain slice of an injected mouse is shown on the right panel, with ChrimsonR-tdTomato shown in red. Scale bar represents $1000 \mu \mathrm{M}$. (B) Representative high magnification images of the CeA in a coronal brain slice of a ChrimsonR-tdTomato injected mouse. ChrimsonR-transduced cells are shown in red and neurons immunostained for PKC $\delta$ in cyan. The merged image is shown on the right panel. Lower insets depict higher magnification images of the areas delineated by the white box in the upper images. White arrowheads highlight representative transduced cells that are also positive for PKCס. Scale bars represent $100 \mu \mathrm{M}$ for low magnification and $10 \mu \mathrm{M}$ for high magnification images. (C) Summary diagram illustrating CeA-PKCס neuron efferent projections within the brain. Forebrain regions are shown in yellow, hypothalamic structures in red, thalamus in green, midbrain in blue and pons in fuchsia. The thickness of the arrows depict the density of labeling (sparse, moderate or dense). (D) Low magnification representative images of brain regions with axonal terminals from CeA-PKC $\delta$ cells. Insets in each image are high magnification images depicting axonal terminals within the regions delineated by the boxes in the respective low magnification images. Scales are $1000 \mu \mathrm{M}$ for low magnification images and $20 \mu \mathrm{M}$ for high magnification images. bed nucleus of stria terminalis medial (BNST-MA); bed nucleus of stria terminalis fusiform nucleus (BNST-Fu); extended amygdala (EA); substantia innominate (SI); lateral preoptic area (LPO); globus pallidus (GP); lateral hypothalamus (LH); Subthalamic nucleus (STh); Zona incerta (ZI); Parasubthalamic nucleus (PSTh); periaqueductal grey (PAG); substantia nigra (SNR); ventral tegmental area (VTA); pedunculopontine tegmental nucleus (PTg); laterodorsal tegmental nucleus (LDTg); reticular formation (mRT); lateral parabrachial (LPB); locus coeruleus (LC). See Figure1 - figure supplement 1. 
bioRxiv preprint doi: $h t t p s: / / d o i . o r g / 10.1101 / 2021.03 .10 .434888$; this version posted March 12,2021 . The copyright holder for this preprint (which was not certified by peer review) is the author/funder, who has granted bioRxiv a license to display the preprint in perpetuity. It is made available under aCC-BY 4.0 International license.

Table 1. CeA-PKCס Neuronal Efferent Targets

\begin{tabular}{llccccc} 
Area & Abbreviations & $\begin{array}{c}\text { ET 987 } \\
\text { (ChrimsonR) }\end{array}$ & $\begin{array}{c}\text { ET 903 } \\
\text { (ChrimsonR) }\end{array}$ & $\begin{array}{c}\text { ET832 } \\
\text { (mCherry) }\end{array}$ & $\begin{array}{c}\text { ET835 } \\
\text { (mCherry) }\end{array}$ & $\begin{array}{c}\text { Allen Brain Atlas } \\
\text { (EGFP) }\end{array}$ \\
\hline
\end{tabular}

\section{Striatum and Basal Forebrain}

\begin{tabular}{|c|c|c|c|c|c|c|}
\hline $\begin{array}{l}\text { Bed nucleus of stria } \\
\text { terminalis }\end{array}$ & BNST & +++ & +++ & +++ & +++ & +++ \\
\hline Globus pallidus & GP & + & + & ++ & + & + \\
\hline Extended amygdala & EA & +++ & +++ & +++ & $+/++$ & +++ \\
\hline Central amygdala & $\mathrm{CeA}$ & +++ & +++ & ++ & $+/++$ & ++ \\
\hline Substantia innominata & SI & ++ & ++ & ++ & $+/++$ & ++ \\
\hline
\end{tabular}

\section{Thalamus}

\begin{tabular}{|c|c|c|c|c|c|c|}
\hline Subthalamic nucleus & STh & ++ & + & + & - & - \\
\hline Zona Incerta & ZI & ++ & + & ++ & - & + \\
\hline $\begin{array}{l}\text { Para subthalamic } \\
\text { nucleus }\end{array}$ & PSTh & ++ & ++ & $+/++$ & + & - \\
\hline \multicolumn{7}{|l|}{ Hypothalamus } \\
\hline lateral preoptic area & LPO & + & + & $+/++$ & + & + \\
\hline Lateral hypothalamus & LH & + & + & $+/++$ & + & + \\
\hline \multicolumn{7}{|l|}{ Midbrain } \\
\hline $\begin{array}{l}\text { Ventral tegmental } \\
\text { area }\end{array}$ & VTA & $+/++$ & + & $+/++$ & - & - \\
\hline Substantia nigra & SN & ++ & $+/++$ & ++ & + & - \\
\hline $\begin{array}{l}\text { Pedunculopontine } \\
\text { tegmental nucleus }\end{array}$ & PTg & + & + & ++ & - & - \\
\hline $\begin{array}{l}\text { Laterodorsal tegmental } \\
\text { nucleus }\end{array}$ & LDTg & + & + & - & + & - \\
\hline Periaqueductal grey & PAG & $+/++$ & + & - & + & - \\
\hline Reticular formation & $\mathrm{mRT}$ & $+/++$ & + & $+/++$ & $+/++$ & - \\
\hline \multicolumn{7}{|l|}{ Pons } \\
\hline Lateral parabrachial & LPB & +++ & +++ & $++/+++$ & + & - \\
\hline Locus coeruleus & LC & + & + & ++ & + & - \\
\hline
\end{tabular}

Table 1. Semi-quantitative analysis of the density of axonal terminals in brain regions from 5 PKC $\delta$-Cre mice stereotaxically injected with a cre-dependent adeno-associated virus anterograde tracer (ChrimsonR, mCherry, EGFP) into the CeA. Rightmost column is from experiment 265945645 of the Mouse Brain Connectivity Atlas of the Allen Brain Institute (http://connectivity.brain-map.org/). - no expression; + sparce; ++ moderate; +++ dense 
experiments. Differences in the size and rostrocaudal distributions of the injections, however, might explain the differences observed in efferent projections between brains.

\section{CeA-PKC $\delta$ neurons send monosynaptic inhibitory projections to the Zona Incerta}

The $\mathrm{ZI}$ was among the regions identified as an efferent target of CeA-PKC $\delta$ neurons in our anatomical experiments (Figure 1C). This subthalamic brain region was of interest because previous studies have shown that reduced activity in GABAergic neurons of the ZI correlates with pain-related behaviors (Masri, et al., 2009; Moon et al., 2016; Moon and Park, 2017; Hu et al., 2019). Given that CeA-PKC $\delta$ neurons are GABAergic and are activated in the context of pain (Wilson et al., 2019), we hypothesized that pain-related inhibition of the $\mathrm{ZI}$ is mediated by injury-induced activation of CeA-PKC $\delta$ neurons (Figure 1E). We began to test this hypothesis by first validating the projections from CeA-PKCס neurons to the $\mathrm{Zl}$ using a retrograde tracer approach where the retrograde tracer cholera toxin B (CTB), conjugated with Alexa Fluor 647, was stereotaxically injected into the ZI of Prkcd-cre::Ai9 mice (Figure 2A). As illustrated in Figure 2B, the retrograde tracer CTB-647 was readily detected in CeA-PKC $\delta$ neurons after injection into the $\mathrm{ZI}$, validating the existence of an anatomical pathway from CeAPKCס neurons to the ZI.

To characterize the functional connectivity between CeA-PKC $\delta$ neurons and $\mathrm{ZI}$ GABAergic neurons, we stereotaxically injected AAV-hsyn-ChR2-EYFP into the CeA of VGAT-cre::Ai9 mice and performed patch-clamp recordings in acute brain slices containing the $\mathrm{ZI}$ (Figure 3A). The stable expression of ChR2-EYFP in the $\mathrm{CeA}$ is indicated by the presence of green fluorescent signal in the peri-somatic region of VGATpositive neurons in CeA. Consistent with our anatomical findings, tracing of the EYFP- 

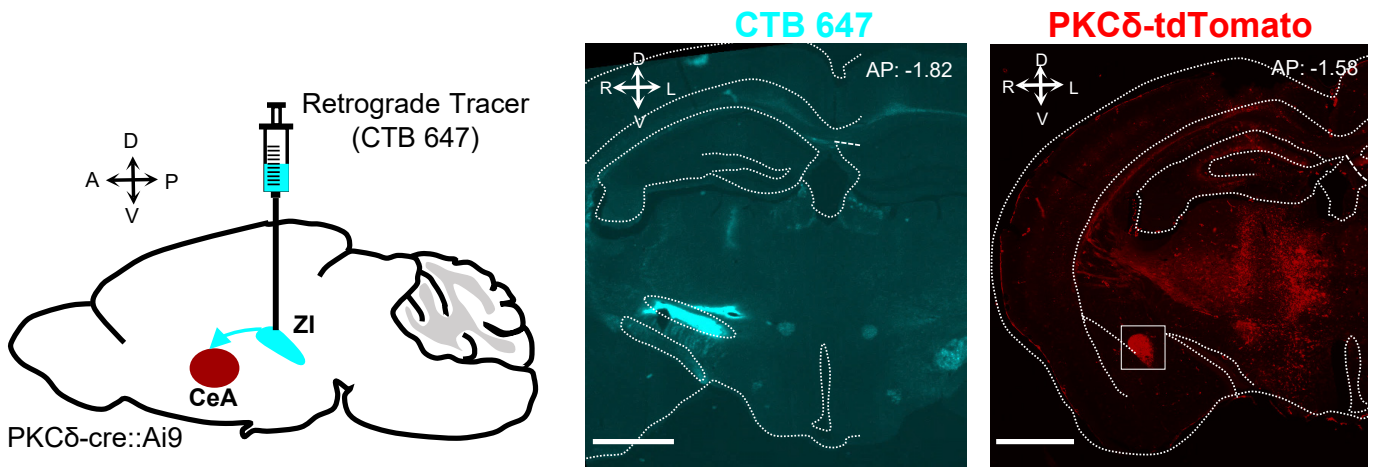

B
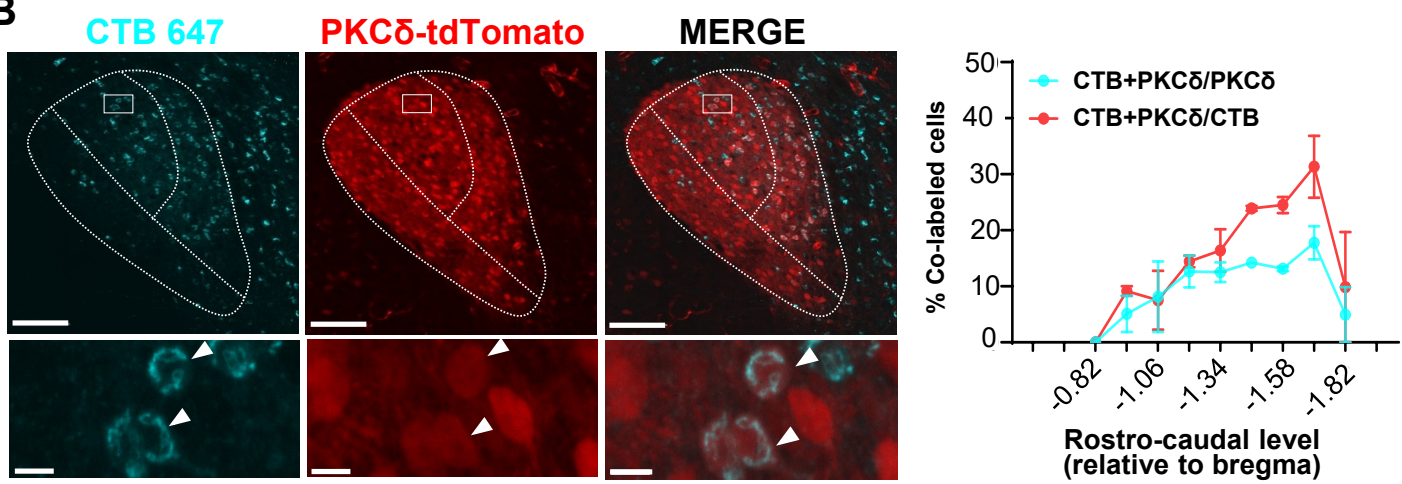

Figure 2. Retrograde tracing of CeA-PKCD projections to the Zl. (A) Schematic drawing of experimental approach (left panel). Cholera toxin B (CTB-647) was injected into the ZI of a PKCठ-cre::Ai9 mouse brain. A representative coronal brain slice depicting the focal injection of CTB-647 (cyan) into the $\mathrm{Zl}$ is shown in the middle panel. A representative coronal brain slice containing the $\mathrm{CeA}$ is shown in the right panel. PKC $\delta$-tdTomato cells are shown in red. The white square delineates the area magnified in panel B. Scale bar represents $1000 \mu \mathrm{M}$. (B) Representative high magnification images of the CeA in a PKC $\delta$-cre::Ai9 mouse injected with CTB-647 in the Zl. CTB-positive cells are shown in cyan and PKCס-tdTomato cells in red. The merged image is shown on the right. Lower insets show higher magnification images of the area delineated by the white squares in the top panel. Arrowheads highlight cells that are positive for CTB and PKC $\delta$-tdTomato. Scale bars represent $100 \mu \mathrm{M}$ (top panel) and $10 \mu \mathrm{M}$ (bottom panel). The mean \pm SEM percentage of cells co-labeled for PKC $\delta$ and CTB as a function of the rostro caudal level is shown on the right ( $\mathrm{n}=2$ mice, 8 slices per mouse). 
153 labeled axonal terminals revealed moderate labeling in both VGAT-positive and VGAT-

154 negative $\mathrm{ZI}$ neurons. Optogenetic stimulation of ChR2-expressing CeA terminals in the ZI

155 with blue light further showed robust inhibitory post-synaptic currents in $60 \%$ VGATpositive (12 out of 20 cells) and 53\% VGAT-negative (8 out of 15 cells) ZI neurons (Figure

3B). These optically evoked postsynaptic responses occurred in the presence of TTX and 4-AP, demonstrating that the inputs from the CeA to the $\mathrm{ZI}$ are monosynaptic. context of pain (Masri et al., 2009; Moon et al., 2016; Moon and Park 2017; Hu et al., 2019). To establish a causal link between reduced activity of ZI-GABAergic neurons and pain-related behaviors, we utilized a chemogenetic approach coupled with a battery of pain behavioral assays to measure tactile and thermal sensitivity in naïve mice as well as in mice with the sciatic nerve cuff model of neuropathic pain. Whole-cell current-clamp recordings in acute $\mathrm{ZI}$ slices prepared from VGAT-cre mice stereotaxically injected with a cre-dependent AAV encoding the inhibitory designer receptors exclusively activated by designer drugs (Gi DREADD) hM4Di into the ZI was used to validate hM4Di-mediated inhibition of ZI neurons (Figure 4A). As illustrated in Figure 4A, bath application of CNO $(10 \mu \mathrm{M})$ significantly inhibited firing responses in hM4Di-transduced neurons, with no

171 measurable effect observed in response to bath application of the saline vehicle control.

172 Histological verification of injection sites at the end of the experiments further 173 demonstrated that transduction of hM4Di-mCherry was restricted to the ZI (Figure 4B 174 and Figure 4 - figure supplement 1 ). The numbers and rostrocaudal distribution of 175 transduced cells within the $\mathrm{Zl}$ of mice stereotaxically injected with hM4Di-mCherry was 
A
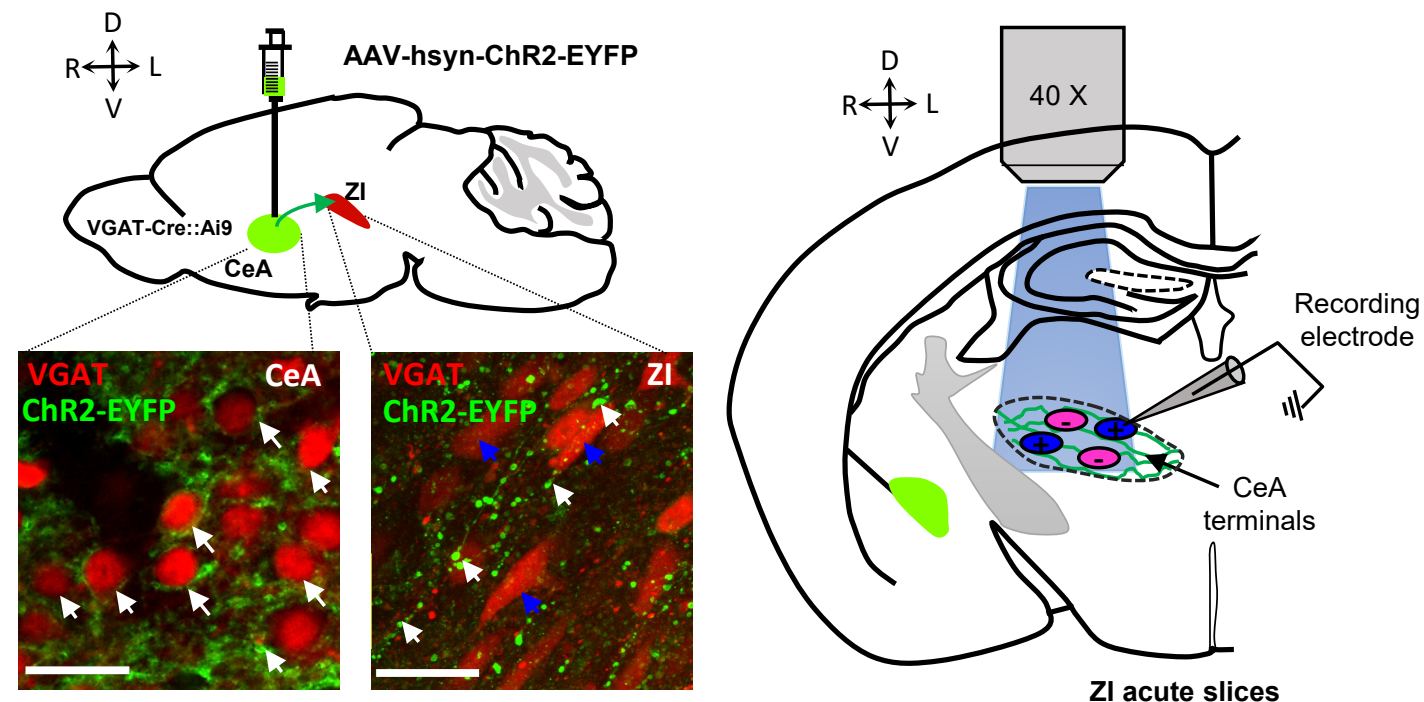

B
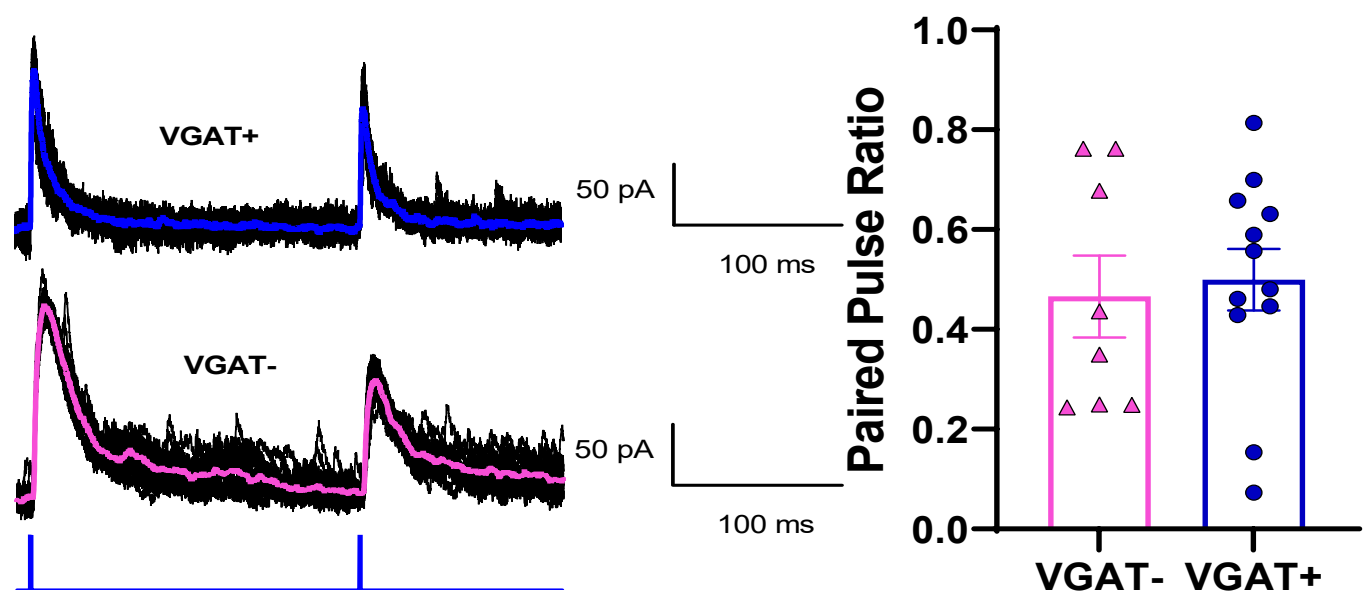

Figure 3. Optogenetic activation CeA terminals in the ZI results in robust inhibitory postsynaptic responses. (A) Schematics of the experimental approach. VGAT-cre::Ai9 mice were stereotaxically injected with AAV-hsyn-hChR2-EYFP into the CeA. After 4 weeks, ChR2-EYFP is expressed in CeA neurons (perisomatic, white arrows, lower left panel) and in the $\mathrm{Zl}$ (CeA terminals, white arrows, lower right panel) in proximity to VGAT positive (blue arrows) cells. Scale bars are $20 \mu \mathrm{M}$. Schematic diagram for ex-vivo whole-cell recordings in acute Zl brain slices is shown in the right panel. Responses of VGAT-positive (blue) and VGAT-negative (magenta) cells are recorded upon optically activating ChR2-expressing CeA terminals. (B) Representative traces showing evoked inhibitory postsynaptic currents in ZI VGAT-positive and VGAT-negative neurons in response to paired pulse stimulation of CeA terminals ( $5 \mathrm{~ms}$ duration, $200 \mathrm{~ms}$ inter stimulus interval). The mean $+/$ - SEM paired pulse ratio is shown on the right panel $(n=8$ VGAT-negative and 12 VGAT-positive cells). 
176 comparable to the numbers and rostrocaudal distribution of VGAT-positive cells in the ZI

177 of VGAT-Cre::Ai9 mice, demonstrating robust transduction efficiency (Figure 4C). related responses to tactile and pressure stimulation of the hindpaws were measured before and after i.p. injection of $\mathrm{CNO}$ to activate the $\mathrm{G}_{\mathrm{i} D R E A D D s}$, or saline as the vehicle control in both cuff and sham mice (Figure 4D). As expected, following cuff implantation on the sciatic nerve, tactile and pressure sensitivity was significantly lower in the hindpaw ipsilateral to cuff implantation compared to the contralateral hindpaw or both hindpaws in sham treated mice (Figures 4E-F). As illustrated in Figures 4E-F, chemogenetic inhibition of VGAT-positive ZI neurons resulted in robust bilateral hypersensitivity to tactile and pressure stimulation in sham mice as well as contralateral hypersensitivity in cuffimplanted mice. Thus, compared to pre-injection values, paw withdrawal thresholds in response to tactile or pinch stimulation were significantly $(p<0.0001)$ reduced bilaterally contralateral to cuff implantation were significantly $(p<0.0001)$ reduced after CNO injection, compared to pre-injection thresholds.

Withdrawal thresholds in the hindpaw ipsilateral to cuff treatment were indistinguishable before and after selective chemogenetic inactivation of VGAT-positive ZI neurons with CNO in hM4Di Zl-injected mice, demonstrating that inhibition of Zl cells does not measurably affect cuff-induced hypersensitivity to tactile and pressure stimulation. Notably, paw withdrawal thresholds in sham mice and the contralateral hindpaw of cuff-implanted mice following the chemogenetic inhibition of ZI VGAT-positive neurons were comparable to the withdrawal thresholds measured in the ipsilateral 


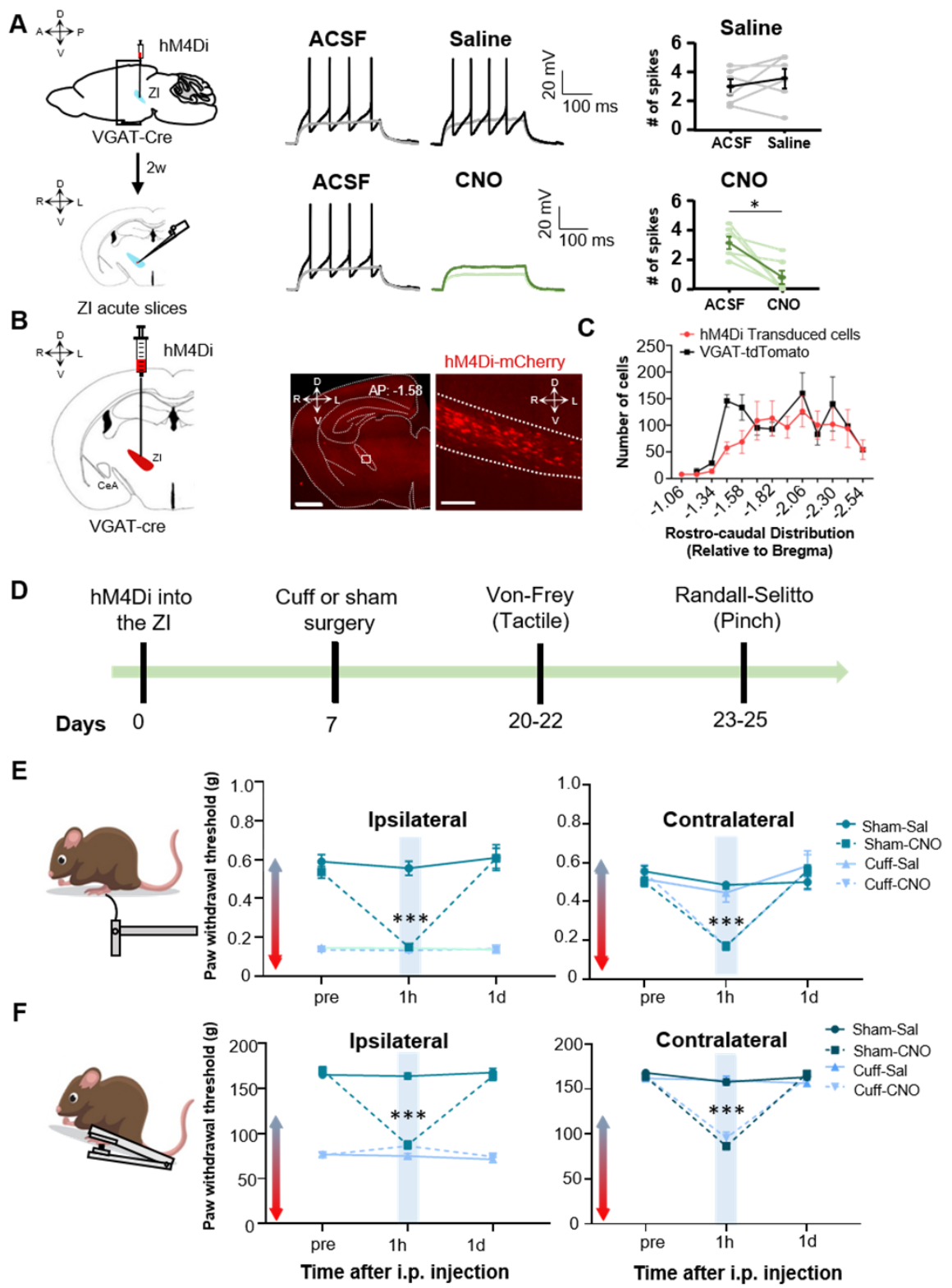

Figure 4. Inhibition of ZI GABAergic neurons is sufficient to induce bilateral tactile and pressure hypersensitivity in uninjured mice. (A) Schematic of the experimental approach. VGAT-Cre mice were stereotaxically injected with AAVDIO-hM4Di-mCherry into the ZI. Current-clamp recordings were obtained from mCherry-positive cells in acute ZI slices 2 weeks after the injection. Representative traces of whole-cell current-clamp recording obtained from $\mathrm{ZI}$ neurons transduced with hM4Di-mCherry before (left) and after (right) bath application of $10 \mu \mathrm{M}$ CNO (lower panel) or vehicle (top panel). Action potential were elicited using 500-ms depolarizing current injection that evoked 2 to 5 action potential before bath the application. The same amplitude of depolarizing current injection was used before and after bath application. Summary graphs depicting the mean \pm SEM number of spikes before and after bath treatment are shown on the right panel ( $n=6$ neurons per treatment; ${ }^{*} p<0.05$ for ACSF vs CNO). (B) Schematic diagram for unilateral stereotaxic injection of AAV-DIO-hM4Di-mCherry in ZI region of VGAT-Cre mice. A representative image of a coronal mouse brain slice from a VGAT-Cre mouse injected with AAV-DIO-hM4Di-mCherry into the $\mathrm{ZI}$ is shown on the middle panel. The area delineated by the white rectangle in the middle panel is shown at higher magnification in the right panel, with mCherry positive cells shown in red. Scale bars represent $1000 \mu \mathrm{m}$ (left) and $100 \mu \mathrm{m}$ (right). (C) Mean \pm SEM number of hM4Di-transduced cells and VGAT-tdTomato labeled cells in the ZI as a function of rostrocaudal level relative to bregma ( $n=11$ mice for hM4Di-transduced neurons; $n=4$ mice for VGATtdTomato neurons). (D) Timeline for behavioral experiments. (E-F) Von Frey (E) and Randall Selitto (F) responses shown as mean \pm SEM paw withdrawal threshold in the ipsilateral (left panel) and contralateral (right panel) hindpaw before, $1 \mathrm{~h}$ and 1 day after CNO or vehicle i.p. injection in cuff or sham mice stereotaxically injected with AAV-DIOhM4Di-mCherry into the Zl. ( $n=8$ mice per treatment; ${ }^{* * *} p<0.0001$ for pre-injections vs $1 \mathrm{~h}$ after CNO in sham-hM4Di CNO treatment for both ipsi and contralateral hindpaws and for cuff-hM4Di-CNO treatment in the contralateral hindpaw; two-way ANOVA followed by Dunnett's multiple comparisons test). See Figure 4 - figure supplement 1. 
199 hindpaw of cuff-implanted mice, demonstrating that inhibition of these neurons is 200 sufficient to elicit hypersensitivity in the absence of injury that resembles the hypersensitivity observed following sciatic nerve cuff implantation. The effects of chemogenetic inhibition of VGAT-positive ZI neurons on tactile and pressure sensitivity were transient, as paw withdrawal thresholds in all treated animals returned to pre204 injection values 1 day following the CNO injections. Importantly, tactile and pressure sensitivity was unaltered in saline-injected mice, demonstrating that the CNO-induced effects were not due to handling or hM4Di expression (Figures 4E-F).

sham treated mice. Behavioral responses to cold and heat stimulation of the hindpaws,

217 in all animals tested. Taken together, the results from these chemogenetic experiments

218 demonstrate that inhibition of VGAT-positive neurons in the $\mathrm{ZI}$ is sufficient to induce

219 hypersensitivity in the absence of injury in a modality-specific manner. 
A

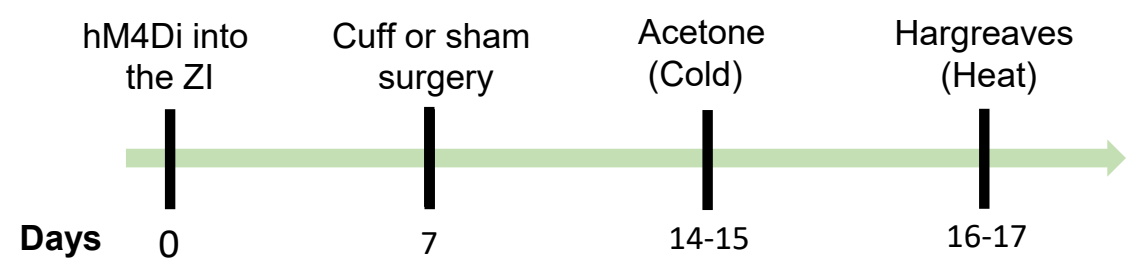

B
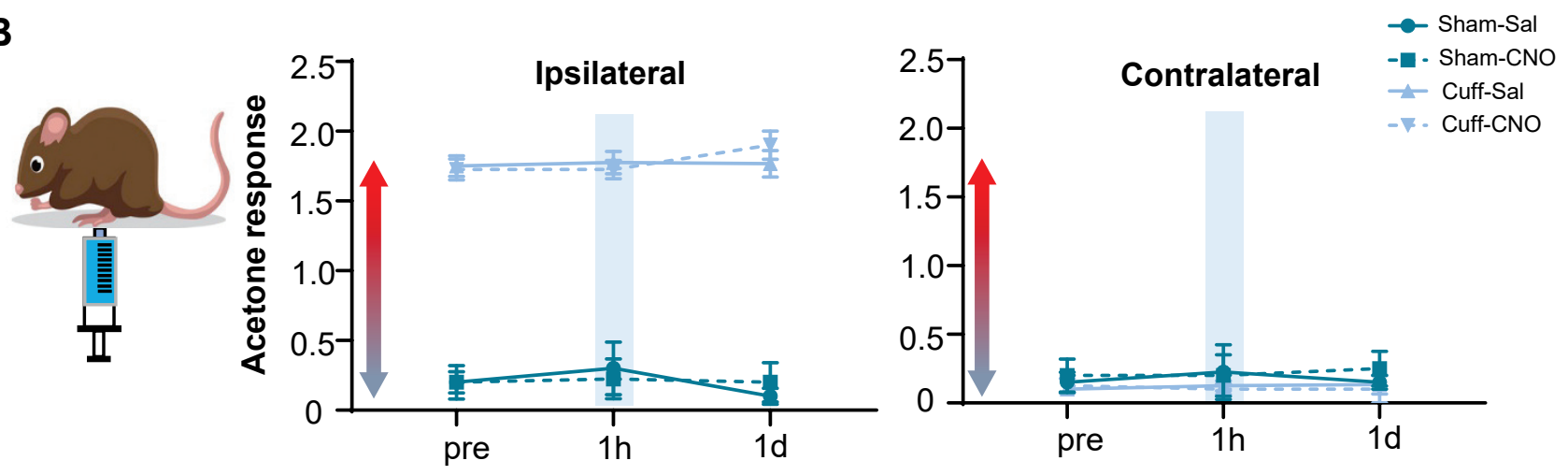

C

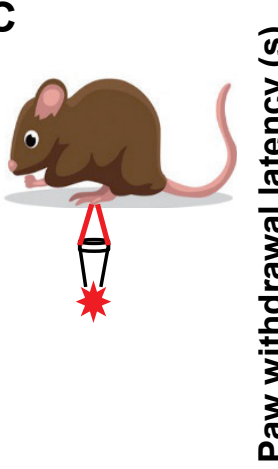

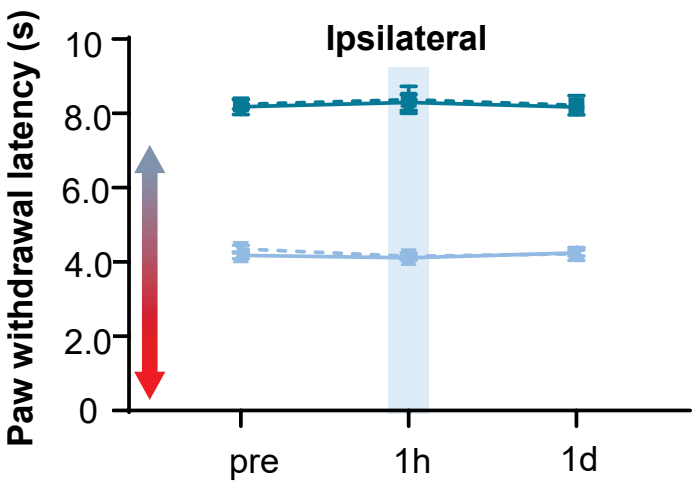

Time after i.p. injection

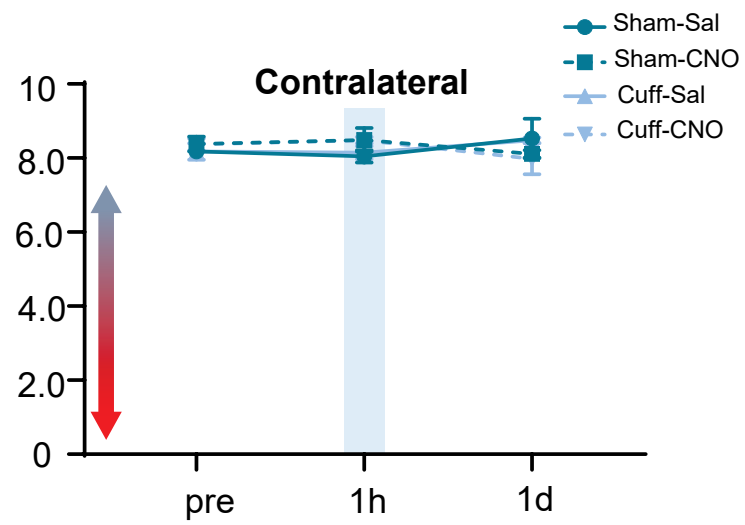

Time after i.p. injection

Figure 5. Cuff-induced thermal hypersensitivity is unaltered by chemogenetic inhibition of ZI-VGAT cells. (A) Timeline for behavioral experiments. (B-C) Mean \pm SEM acetone response (B) or paw withdrawal latency obtained from Hargreaves test (C) in the ipsilateral (left panel) and contralateral (right panel) hindpaw before, $1 \mathrm{~h}$ and 1 day after $\mathrm{CNO}$ or vehicle i.p. injection in cuff or sham mice stereotaxically injected with AAV-DIO-hM4Di-mCherry into the ZI. ( $n=8$ mice per treatment; no significant differences were observed in any treatment; two-way ANOVA followed by Dunnett's multiple comparisons test). 
The next set of experiments aimed to determine whether activation of GABAergic approach coupled with behavioral assays to measure tactile and heat sensitivity in mice

225 following the implantation of a sciatic nerve cuff to model neuropathic pain. Histological

226 verification of the injection sites at the end of the experiments demonstrated that

227 transduction of hM3Dq-mCherry and control-mCherry was restricted to the Zl (Figure 6A and Figure 6 - figure supplement 1 and 2). The numbers and rostrocaudal distribution of transduced cells within the $\mathrm{ZI}$ were comparable in mice injected with hm3Dq-mCherry and control-mCherry and show robust transduction efficiency that is localized to the $\mathrm{ZI}$

(Figure 6B). CNO-mediated activation of neurons in VGAT-Cre mice stereotaxically injected with AAV8-DIO-hM3Dq-mCherry into the ZI was validated with immunohistochemical monitoring of c-Fos, the product of an immediate early gene that is commonly used as a marker of neuronal activity (Figure 6C). As illustrated in Figure 6D, i.p. injection of CNO resulted in robust c-Fos expression in the ZI of VGAT-Cre animals injected with hM3Dq, compared to the c-Fos expression observed in the $\mathrm{Zl}$ of salineinjected control mice that also expressed hM3Dq or CNO-injected control VGAT-Cre mice stereotaxically injected with the control virus (AAV8-DIO-mCherry). Quantification of ZI

239 cells co-expressing c-Fos and mCherry further confirms that c-Fos expression in hM3Dqtransduced cells is significantly $(p<0.05)$ higher in CNO-treated mice than it is in salinetreated mice or in mCherry-transduced neurons from CNO-treated mice (Figure 6E). responses to pressure (pinching) stimulation of the hindpaws was measured before and 244 after i.p. injection of CNO or saline in cuff and sham animals (Figure 6F). CNO-mediated 
A

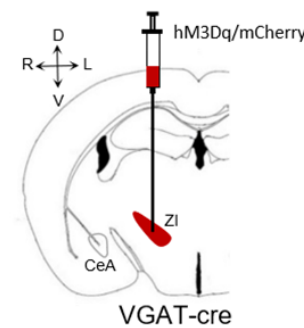

C
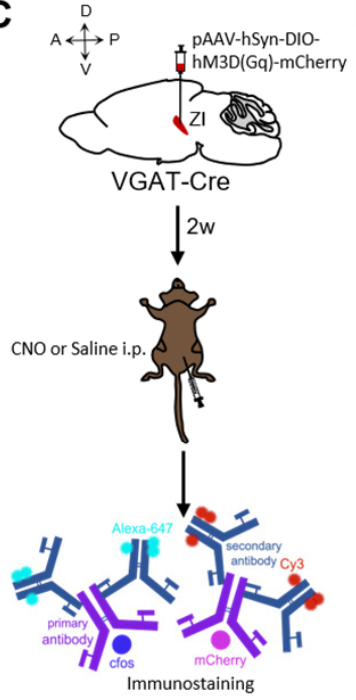

E

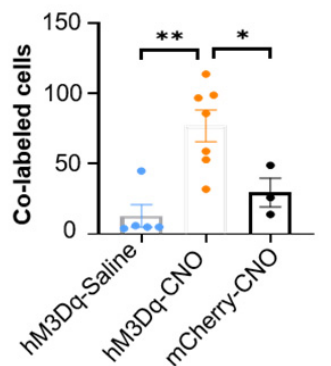

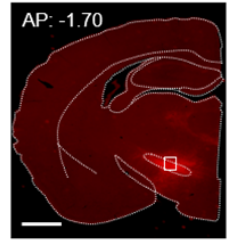
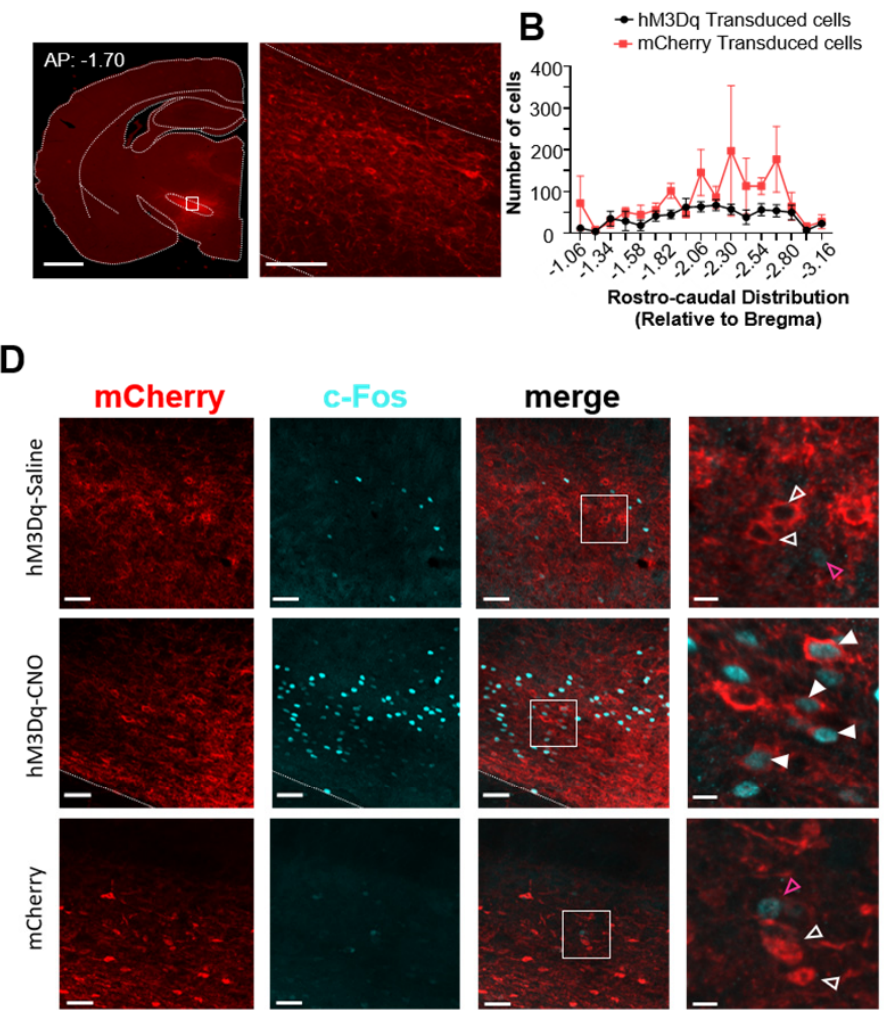

$\mathbf{F}$
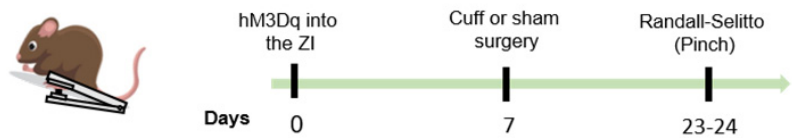
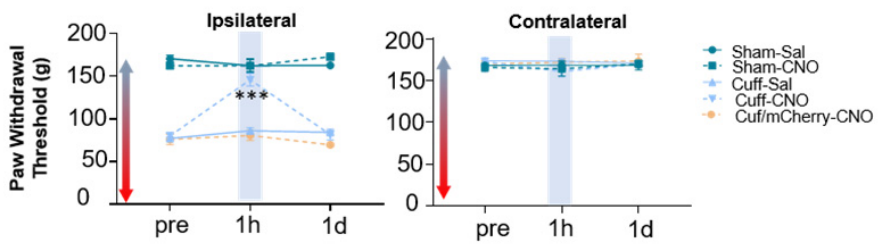

Figure 6. Activation of $\mathrm{ZI}$ GABAergic cells reverses cuff-induced hypersensitivity to pinch stimulation. (A) VGAT-Cre mice were injected with AAV-DIO-hM3Dq-mCherry or AAV-DIO-mCherry into the Zl. Low magnification representative image of a coronal brain slice shows the site of virus injection in red. The area delineated by the white rectangle is shown at higher magnification in the right image. Scale bars are $1000 \mu \mathrm{m}$ for low magnification and $100 \mu \mathrm{m}$ for high magnification images (B) Quantification of ZI cells transduced with $\mathrm{hM} 3 \mathrm{Dq}$ and mCherry is shown as mean \pm SEM $(n=17$ mice for $h M 3 D q$ transduced group and 7 mice for mCherry group). (C) c-Fos experimental timeline. (D) Representative images of coronal brain slices containing the ZI of VGAT-Cre mice injected with AAV-DIO-hM3Dq-mCherry (top and middle panels) or AAV-DIO-mCherry (bottom) into the $\mathrm{ZI}$ and i.p. treated with $\mathrm{CNO}$ (middle and bottom panels) or saline (top panel). Top panel shows images from mouse with mCherry (Control virus) injected in Zl. mCherry expression is shown in red and immunostaining for c-Fos in cyan. The merged images are shown in the rightmost panels. White boxes delineate the areas magnified on the right panel. Magenta open arrowheads point to cells that are positive for cFos only; white open arrowheads point to cells that are positive for mCherry only; solid arrowheads point to cells that are positive for both mCherry and c-Fos. Scale bars are $50 \mu \mathrm{m}$ (low magnification) and $10 \mu \mathrm{m}$ (high magnification). (E) Mean \pm SEM numbers of c-Fos and mCherry transduced co-labeled cells per condition. ( $n=3-7$ mice per condition; ${ }^{* *} p<0.01$, * $p<0.05$; One-way ANOVA followed by Tukey's multiple comparisons test). (F) Randall Selitto responses are shown as mean \pm SEM paw withdrawal threshold in the ipsilateral (left panel) and contralateral (right panel) hindpaw before, $1 \mathrm{~h}$ and 1 day after CNO or vehicle i.p. injections in cuff or sham mice stereotaxically injected with AAV-DIO-hM3Dq-mCherry or AAV-DIO-mCherry into the ZI ( $n=6-8$ mice per condition; ${ }^{* * *} p<0.0001$ for pre-injections vs $1 \mathrm{~h}$ after CNO in cuff-hM3Dq CNO treatment; two-way ANOVA followed by Dunnett's multiple comparisons test). See Figure 6 - supplemental figures 1 and 2. 
245 activation of VGAT-positive $\mathrm{ZI}$ neurons led to significant $(p<0.0001)$ reversal of cuff-

246 induced hypersensitivity in the hindpaw ipsilateral to cuff implantation, while no

247 measurable effects were seen in saline-injected or CNO-injected mCherry-control mice.

248 The effects of activation of VGAT-positive ZI neurons were specific to nerve injury as

249 withdrawal thresholds in sham treated mice or the hindpaw contralateral to sciatic nerve

250 cuff were comparable between groups. As illustrated in Figure 7, reversal of cuff-induced

251 hypersensitivity was also modality-specific as behavioral responses to cold and heat

252 stimulation of the hindpaws were unaffected by chemogenetic activation of VGAT-positive

253 neurons in the ZI. Taken together, these results demonstrate that activation of VGAT-

254 positive neurons in the $\mathrm{Zl}$ reverses cuff-induced pain hypersensitivity in a modality-

255 specific manner.

\section{Discussion}

Signals for persistent neuropathic pain in distant parts of the body are perceived induced increases in the activity of CeA-PKCס neurons, for example, have been shown to contribute to behavioral hypersensitivity (Wilson et al., 2019). The neural circuits involved in modulation of nociceptive behaviors by CeA-PKCס cells, however, remains

265 unknown. In the present study, we identified 17 long-range efferent projections from CeA-

266 PKCס neurons, including the $\mathrm{ZI}$ which has been previously reported to be involved in pain

267 processing. We further demonstrated that the $\mathrm{ZI}$ receives strong monosynaptic inhibitory 268 inputs from CeA-PKCס neurons and that chemogenetic manipulation of the activity of 
A

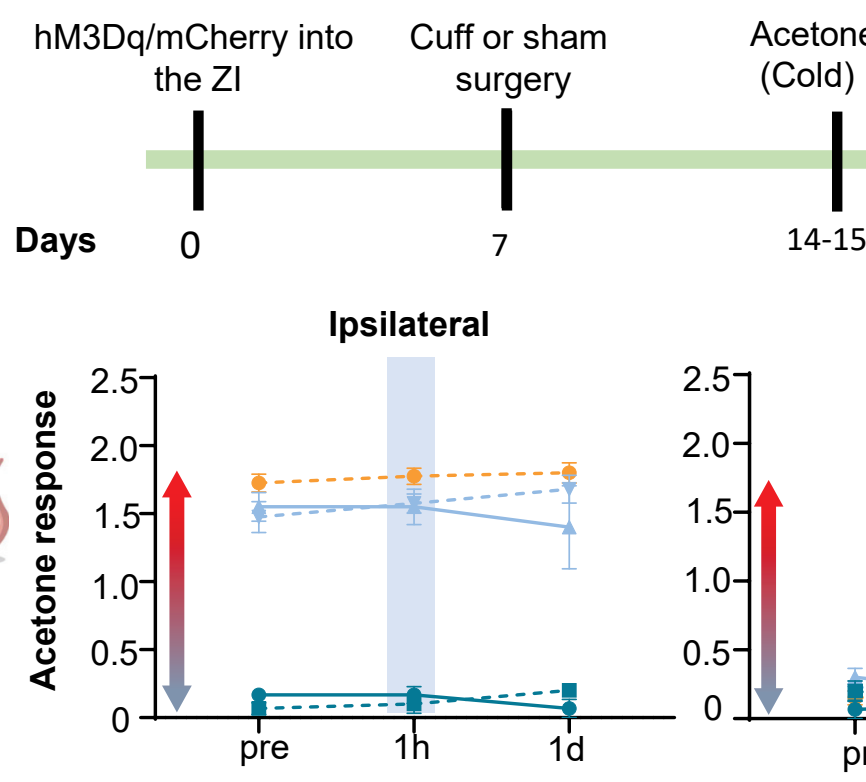

Acetone

(Cold)

Hargreaves

(Heat)

14-15

16-17

B
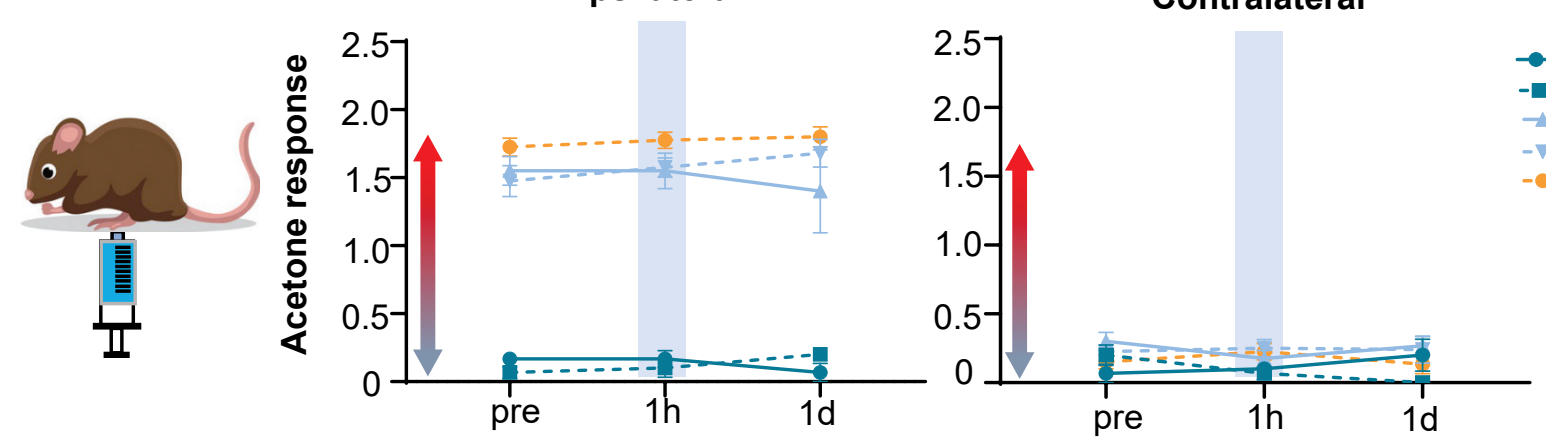

C
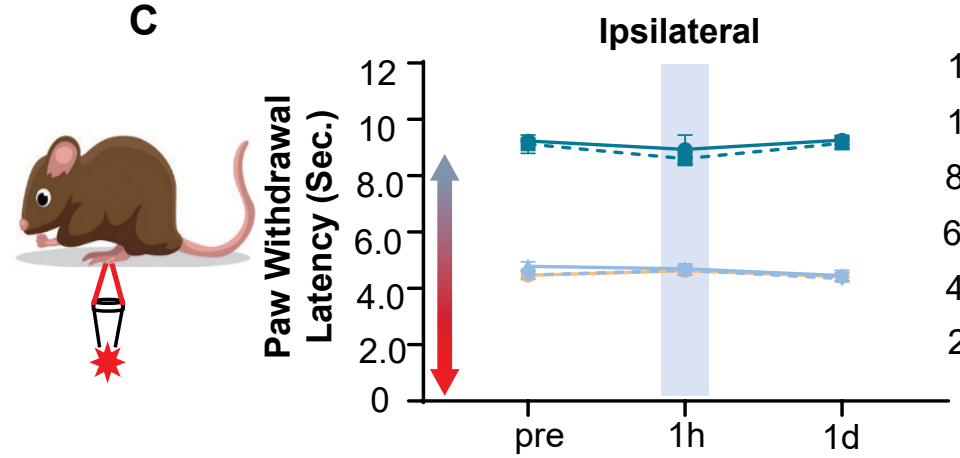

Time after i.p. injection

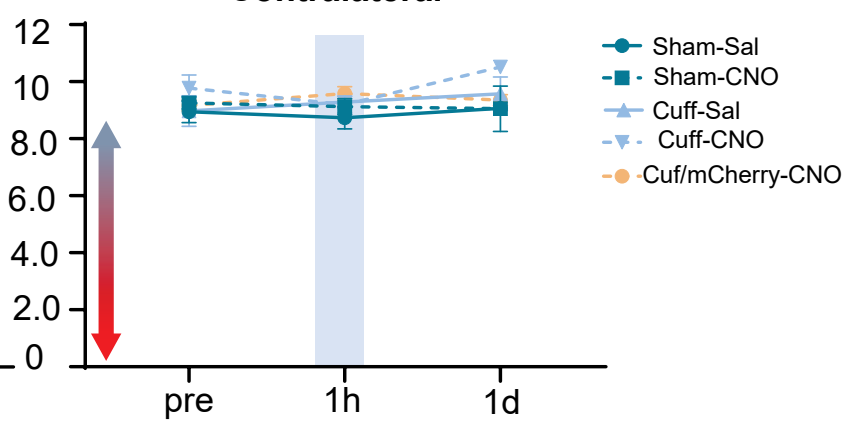

Time after i.p. injection

Figure 7. Cuff-induced thermal hypersensitivity is unaltered by chemogenetic activation of ZI-VGAT cells. (A) Experimental timeline. (B-C) Mean \pm SEM acetone response (B) or paw withdrawal latency obtained from Hargreaves test (C) in the ipsilateral (left panel) and contralateral (right panel) hindpaw before, $1 \mathrm{~h}$ and 1 day after CNO or vehicle i.p. injection in cuff or sham mice stereotaxically injected with AAV-DIO-hM3Dq-mCherry or AAV-DIO-mCherry into the $\mathrm{ZI}$. ( $\mathrm{n}=4-8$ mice per treatment; no significant differences were observed in any treatment; two-way ANOVA followed by Dunnett's multiple comparisons test). 
GABAergic neurons in the ZI bidirectionally modulates pain-related behavioral outputs in a modality-specific manner. Altogether, our results identify the $\mathrm{ZI}$ as a functional anatomical target of the $\mathrm{CeA}$ that contributes to the modulation of pain-related behaviors.

\section{Proposed Model}

Previous studies have shown that spontaneous firing rates and somatosensoryevoked neuronal responses decrease in the $\mathrm{Zl}$ in a rodent model of central pain syndrome, subsequently disinhibiting the posterior thalamus (PO) (Masri et al., 2009). In the $\mathrm{CeA}$, previous studies have demonstrated that CeA-PKC $\delta$ neurons are activated in a model of neuropathic pain and drive behavioral hypersensitivity (Wilson et al., 2019). Given the results presented here demonstrating a strong monosynaptic inhibitory input from the $\mathrm{CeA}$ to the $\mathrm{ZI}$ (Figure 3), in combination with the $\mathrm{ZI}$ retrograde tracer experiments showing high colocalization of retrograde tracer uptake in CeA-PKC $\delta$ neurons (Figure 2) and the behavioral experiments showing that inhibition of ZI GABAergic neurons results in robust bilateral behavioral hypersensitivity (Figure 4), we propose a model where injury-induced activation of CeA-PKCס neurons inhibits ZI-GABAergic cells, subsequently leading to behavioral hypersensitivity (Figure 8).

\section{CeA inputs to Zona Incerta}

Our optogenetic-assisted circuit mapping experiments showed that both VGATpositive and VGAT-negative $\mathrm{ZI}$ cells respond to optogenetic stimulation of CeA terminals in the ZI (Figure 3). Similarly, our anatomical experiments show retrograde uptake in both PKC $\delta$-positive and PKC $\delta$-negative cells in the CeA when the retrograde tracer was injected in the ZI (Figure 2). These results are consistent with previous studies that have shown that somatostatin-expressing neurons in the $\mathrm{CeA}$, which have virtually no overlap 


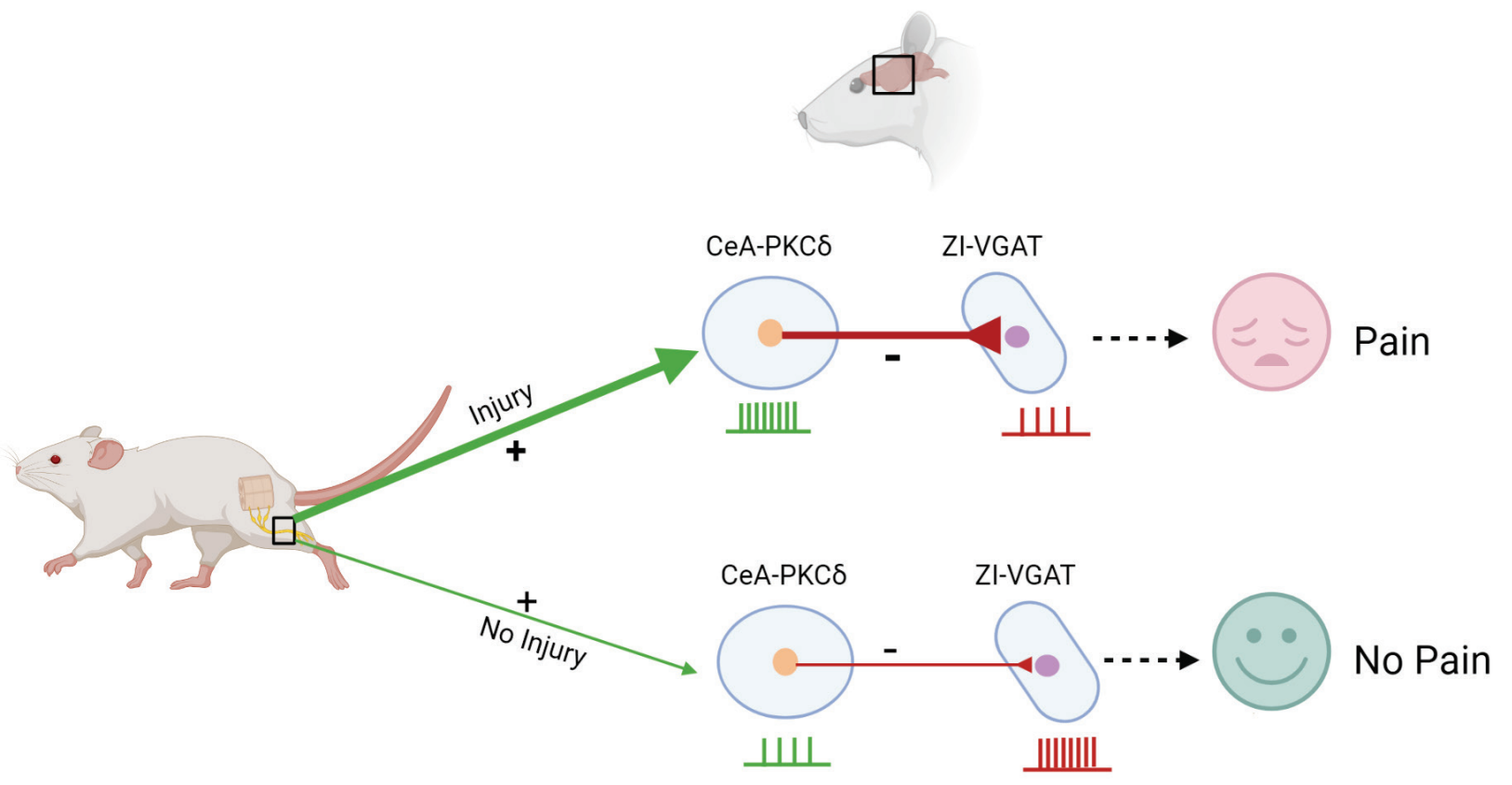

Figure 8. Proposed model for modulation of pain-related behaviors in the Zl. Schematic drawing showing ZI-VGAT neurons receive monosynaptic inhibitory inputs from CeA-PKCס neurons and modulate pain-related behaviors. After injury, activation of CeA-PKCס neurons inhibits ZI-VGAT neurons, subsequently leading to behavioral hypersensitivity. 
292 with CeA-PKCס neurons, also project to the Zl (Zhou et al., 2018). Interestingly, in this

293 study, Zhou and colleagues further demonstrated that efferent inputs from somatostatin-

294 expressing neurons in the CeA selectively target parvalbumin-expressing neurons in the

$295 \mathrm{Zl}$ and contribute to fear memory. Together, these results suggest that genetically distinct

296 CeA neurons might target neurochemically distinct subpopulations of neurons in the $\mathrm{ZI}$

297 and that cell-type-specific CeA-ZI pathways might differentially contribute to the 298 modulation of pain-related behaviors.

\section{Modulation of pain-related behaviors in the $\mathrm{ZI}$}

In the present study, we show that chemogenetic manipulation of the activity of

specific manner (Figures 4-7). Thus, we observe bilateral hypersensitivity to tactile (but of nerve or tissue injury (Figures 4-5). In contrast, chemogenetic activation of VGATinhibition of the $\mathrm{ZI}$ are similar to those observed after chemogenetic activation of the CeA-

311 (Wilson et al., 2019). Together, these results support our proposed model where injury312 induced activation of CeA-PKCD neurons serves as the source of inhibition of $\mathrm{Zl}$, 313 contributing to behavioral hypersensitivity (Figure 8). 

while inhibition of CeA-PKCס neurons reversed cuff-induced hypersensitivity to tactile, cold and heat hypersensitivity (Wilson et al., 2019), chemogenetic activation of Zl GABAergic neurons only reversed cuff-induced pinch, but not cold or thermal, hypersensitivity (Figure 6-7). Previous studies have shown that responses of spinoparabrachial neurons are modality-specific (Hachisuka et al., 2020). Our results

321 raise the interesting possibility that subpopulations CeA-PKC $\delta$ neurons might also be recruited in a modality-specific manner, with CeA-PKCס neurons that project to the ZI selectively responding to tactile but not thermal stimulation.

Inconsistencies with previous studies and potential cell-type-specificity in the ZI treatment were unaltered by chemogenetic activation of VGAT-positive ZI neurons in the 327 present study. This contrasts with previous reports, where optogenetic activation of VGAT-positive ZI neurons decreased baseline responses to tactile stimulation (Hu et al., 2019). Previous studies have demonstrated that modulation of behavioral output by optogenetic stimulation is dependent on the frequency and pattern of the stimulation used

331 for the experiments (Padilla-Coreano et al., 2019). It is therefore possible that the 332 differences in the results from our chemogenetic study and the previous optogenetic study 333 might stem from different levels of neuronal activation by these various techniques. the contribution of a different subpopulation of ZI neurons- those expressing parvalbumin 
337 (Wang et al., 2020). Interestingly, this study demonstrated that parvalbumin-positive neurons in the $\mathrm{ZI}$ show an opposite electrophysiological phenotype and function in the modulation of pain-related behaviors than what we and others observe for the VGATpositive ZI neurons. Thus, while the present and previous studies show that inhibition of VGAT-positive neurons drives behavioral hypersensitivity, activation of parvalbumin-

342 positive $\mathrm{ZI}$ neurons have been previously shown to also promote behavioral hypersensitivity (Wang et al., 2020). These results are particularly interesting given that

344 other studies have demonstrated that parvalbumin-positive ZI neurons receive inputs 345 from somatostatin-expressing CeA neurons (Zhou et al., 2018), which have been previously shown to be inhibited in the context of pain (Wilson et al., 2019). inhibition of somatostatin-expressing neurons in the CeA disinhibits parvalbumin-positive ZI neurons, while injury-induced activation of CeA-PKCס neurons inhibits VGAT-positive ZI neurons, both resulting in behavioral hypersensitivity. Whether injury-induced inhibition of VGAT-positive ZI neurons (by CeA-PKCס neurons) occurs simultaneously and under

352 the same conditions as disinhibition of parvalbumin-positive $\mathrm{ZI}$ neurons (by somatostatinexpressing neurons in the $\mathrm{CeA}$ ) remains to be determined. Future studies to address

354 these important questions directly will provide further insights about the CeA-ZI neuronal 355 connection and the potential cell-type-specific contributions to the modulation of pain356 related behaviors. 
All experiments were approved by the Animal Care and Use Committee of the

361 National Institute of Neurological Disorders and Stroke and the National Institute on

362 Deafness and other Communication Disorders with the guidelines set by the National

363 Institutes of Health $(\mathrm{NIH})$. Adult male mice (8 to 17-weeks old) were used for all

364 experiments. Mice were housed in a vivarium with controlled humidity and temperature

365 under reversed $12 \mathrm{~h}$ light/dark cycle (9 pm to $9 \mathrm{am}$ light) with ad libitum access to food

366 and water. All the behavioral tests were performed during the dark period, between the

367 hours of 10 am and 6 pm. Mice received $100 \mu$ of saline intraperitoneal injection (i.p.)

368 daily and were handled by the experimenter for one week before the start of behavioral

369 and electrophysiological experiments following the cupping method as previously

370 described (Hurst and West, 2010). Following surgeries, mice were housed in pairs and

371 separated by a perforated Plexiglas divider. Heterozygous male or female Prkcd-cre mice

372 (GENSAT-founder line 011559-UCD) were crossed with Ai9 mice (Jackson Laboratories);

373 vesicular GABA transporter Cre mice (Slc32a1-ires-Cre, or VGAT-Cre mice; Jackson

374 Laboratories) were bred as homozygous pairs or crossed with Ai9 mice (Jackson

375 Laboratories). Both the Prkcd-cre and VGAT-Cre mouse lines have been previously

376 validated and shown to express Cre selectively in PKCס+ and GABAergic neurons,

377 respectively (Vong et al., 2011; Wilson et. al., 2019). The presence of cre-recombinase

378 in offspring was confirmed by genotyping using DNA extracted from tail biopsies. The

379 primer sequences (Transnetyx) used for genotyping were

380 TTAATCCATATTGGCAGAACGAAAACG $\quad$ (forward primer) and

381 AGGCTAAGTGCCTTCTCTACA (reverse primer).

382 Stereotaxic Injections in the $\mathrm{CeA}$ and $\mathrm{ZI}$ 
Mice were initially anesthetized with $5 \%$ isoflurane in preparation for the stereotaxic surgery. After induction, mice were head-fixed on a stereotaxic frame (David Kopf Instruments) and $1.5-2 \%$ isoflurane at a flow rate of $0.5 \mathrm{~L} / \mathrm{min}$ was used for the duration of surgery. A hand warmer was used for thermal maintenance during the procedure. Stereotaxic injections were performed using a 32-gauge needle that fits on a $0.5 \mu$ volume Hamilton Neuros syringe. All the injections were performed at a flow rate of $0.1 \mu \mathrm{l} / \mathrm{min}$ and the syringe was left in place for an additional $5 \mathrm{~min}$ after injections to allow for the diffusion of virus and to prevent backflow. \#50459-AAV8) or AAV9-Syn-Flex-ChrimsonR-TdTomato (UNC GTC Vector Core, AV4384G) was microinjected into the right CeA of Prkcd-cre mice. The coordinates relative to bregma were as follows: $\mathrm{AP}-1.4 \mathrm{~mm}, \mathrm{ML}+3.2, \mathrm{DV}-4.8 \mathrm{~mm}$. For retrograde labeling, $0.2 \mu \mathrm{l}$ of Alexa Fluor 647-conjugated cholera toxin subunit B (Invitrogen, C34778) was stereotaxically injected into the right $\mathrm{Zl}$ of Prkcd-cre::Ai9 mice. The coordinates relative to bregma were as follows: AP $-1.9 \mathrm{~mm}, \mathrm{ML}-1.4 \mathrm{~mm}$, DV $-4.75 \mathrm{~mm}$. 398 For optogenetic-assisted circuit mapping experiments, $0.3 \mu \mathrm{l}$ of rAAV2-hSyn399 hChR2(H134R)-EYFP-WPRE-PA (UNC GTC Vector Core, AV6556C) was microinjected 400 into the right CeA of VGAT-Cre::Ai9 mice. The coordinates relative to bregma were as follows: AP -1.25 mm, ML -3.0 mm, DV -4.5 mm. For chemogenetic experiments, $0.15 \mu \mathrm{l}$ 402 of pAAV8-hSyn-DIO-hM4D(Gi)-mCherry (Addgene viral prep \#44362-AAV8; Krashes et al., 2011), pAAV8-hSyn-DIO-hM3D(Gq)-mCherry (Addgene viral prep \#44361; Krashes 404 et al., 2011) or rAAV8-hSyn-DIO-mCherry (Addgene viral prep \#50459-AAV8) were 405 microinjected into the right ZI of VGAT-Cre mice. The coordinates relative to bregma were 
421 behavior tests.

as follows: $\mathrm{AP}-1.7 \mathrm{~mm}, \mathrm{ML}-1.2 \mathrm{~mm}$, DV $-4.7 \mathrm{~mm}$. A minimum of 2 weeks was given between brain injections and behavior testing to allow for efficient viral-mediated transduction. For retrograde, anterograde and optogenetic experiments, we waited a minimum of 4 weeks between the brain injections and the experiments.

\section{Sciatic cuff implantation}

Sciatic cuff implantation surgeries were performed 1 week after the brain surgeries as previously described (Benbouzid et al., 2008). Briefly, mice were anesthetized with $2 \%$ isoflurane at a flow rate of $0.5 \mathrm{~L} / \mathrm{min}$. An incision of about $1-\mathrm{cm}$ long was made in the proximal one third of the lateral left thigh. The sciatic nerve was externalized and stretched using forceps. For the cuff-implanted group, a polyethylene tubing PE20 (2 mm-long, 0.38 mm ID / 1.09 mm OD; Daigger Scientific) was slid onto the sciatic nerve and was then placed back in its location. Similarly, for the comparative sham group, mice went through the same process of sciatic nerve exposure and stretching but no tubing was implanted. After the procedure was complete, the skin above the thigh was closed with wound clips. The mice were subjected to at least one-week recovery period before performing the

\section{Nociceptive Behaviors}

Behavioral experiments were done under red light, during the dark phase, and the experimenter was blind to treatment conditions. Mice were randomized into control and experimental groups. The timeline for the behavioral experiments relative to AAV brain injections were as follows: Acetone test: 14-15 days; Hargreaves test: 16-17 days; VonFrey test: 20-22 days; Randall-Sellito test: 23-25 days. Each test was performed on 2 
428 consecutive days. On each testing day, baseline (pre-injection) measurements were 429 taken. Saline or Clozapine-N-oxide (CNO, Enzo Life Sciences, Farmingdale, NY) was

430 injected i.p. (10 mg/kg body weight for hM4Di and $5 \mathrm{mg} / \mathrm{kg}$ for hM3Dq experiments) and

431 a second measurement (post-injection) was taken 45 min to 1 hour after the i.p. injection.

432 Mice were randomly assigned to saline or CNO on the first day of each test and were

433 tested on the opposite treatment on the second day of the same test. described (Choi Yoon 1994). Briefly, mice were habituated for at least 2 hours in individual ventilated opaque white Plexiglas testing chambers $(11 \times 11 \times 13 \mathrm{~cm})$ on an elevated platform with a floor made of wire mesh. An acetone drop was formed at the top of a 1 $\mathrm{mL}$ syringe and gently touched to the center of the plantar surface of the hind paw ipsilateral or contralateral to sciatic nerve surgery. Nociceptive responses to the acetone

441 drop were evaluated for 60 seconds using a modified 0-2-point system developed by 442 Colburn et al., 2007. According to this scoring system, 0 = rapid, transient lifting, licking, 443 or shaking of the hind paw, which subsides immediately; 1 = lifting, licking, and/or shaking 444 of the hind paw, which continues beyond the initial application, but subsides within 5 s; 2 $445=$ protracted, repeated lifting, licking, and/or shaking of the hind paw. Five trials were 446 performed with $\sim 5$ min between-trial intervals. Data is represented as an average score 447 of 5 stimulations from each hindpaw before, 1 hour and 1 day after drug treatment (CNO 448 or vehicle). 
To evaluate heat hypersensitivity, we used a modified version of the Hargreaves

451

452

453

454

455

456

457

458

459

460

461

462

463

464

465

466

467

468

469

470

471

Method (Hargreaves et. al., 1988) as previously described (Wilson et.al., 2019). On the experiment day, mice were habituated prior to testing for at least 1 hour in individual ventilated opaque white plexiglass testing chambers $(11 \times 11 \times 13 \mathrm{~cm})$ placed on an elevated glass floor maintained at $30^{\circ} \mathrm{C}$. Following habituation, a noxious radiant heat beam was applied through the glass floor (IITC Life Sciences, Woodland Hills, CA) to the center of the plantar surface of the hindpaw (ipsilateral or contralateral to sciatic nerve surgery), until the mouse showed a withdrawal response. A cutoff of 15 seconds latency and 25 active intensity was used in each trial to prevent skin lesions. At least 3 minutes were allowed between consecutive trials. The average of five trials was calculated and used as the threshold for each hindpaw.

\section{Von Frey}

Mechanical hypersensitivity was assessed as the paw withdrawal threshold in response to von Frey filaments (North Coast Medical, Inc. San Jose, CA), as previously described (Wilson et. al., 2019). On each testing day, mice were placed individually in ventilated opaque white Plexiglas testing chambers $(11 \times 11 \times 13 \mathrm{~cm})$ on an elevated mesh platform at least 2 hours before application of stimulus. Mesh floor allowed full access to the paws from below. After the acclimation period, each von Frey filament was applied to the center of the plantar surface of the hindpaw (ipsilateral or contralateral to sciatic nerve surgery) for 2-3 seconds, with enough force to cause slight bending against the paw. This procedure continued for a total of five measurements. The smallest filament that evoked a paw withdrawal response in at least three of five measurements was taken 
472 as the mechanical threshold for that trial. The average of five trials was calculated and

473 used as the threshold value per hindpaw.

474 Randall Selitto Test

The Randall Selitto test was performed to assess the response thresholds to mechanical pressure stimulation (pinch) of the hind paws (Randall and Selitto, 1957) in

477 lightly anesthetized animals. Briefly, mice were anesthetized in $5 \%$ isoflurane in an 478 induction chamber. Subsequently, animals were kept under light anesthesia with $0.5 \%-$ $4791 \%$ isoflurane at a flow rate of $0.5 \mathrm{~L} / \mathrm{min}$. A pinch stimulation of less than $200 \mathrm{~g}$ of force 480 was delivered to the plantar surface of the hindpaw ipsilateral or contralateral to sciatic nerve treatment. Pinch pressure stimulation was applied at approximately 1-minute intervals for 30 minutes. The pressure that triggered withdrawal of paw was recorded in all trials. The data is represented as the average response for all trials for each animal before, 1 hour and 1 day after drug treatment (CNO or vehicle).

Slice Electrophysiology

Acute coronal ZI slices were prepared from brains of VGAT-Cre or VGAT-Cre::Ai9 mice (12-to 18-week-old) 2-3 weeks after stereotaxic injection of AAV8-hSyn-DIO-hM4DimCherry into the ZI or 5-8 weeks after AAV2-hsyn-hChR2(H134R)-EYFP injection into the CeA. Briefly, mice were deeply anesthetized with $1.25 \%$ Avertin anesthesia $(2,2,2-$ tribromoethanol and tert-amyl alcohol in $0.9 \% \mathrm{NaCl} ; 0.025 \mathrm{ml} / \mathrm{g}$ body weight), sacrificed by cervical dislocation and decapitated. The brains were rapidly removed and placed in 492 an ice-cold cutting solution containing (in mM): 110.0 choline chloride, $25.0 \mathrm{NaHCO}_{3}, 1.25$ $493 \mathrm{NaH}_{2} \mathrm{PO}_{4}, 2.5 \mathrm{KCl}, 0.5 \mathrm{CaCl}_{2}, 7.2 \mathrm{MgCl}_{2}, 25 \mathrm{D}$-glucose,12.7 L-ascorbic acid, 3.1 pyruvic 
494 acid, and saturated with $95 \% \mathrm{O}_{2}-5 \% \mathrm{CO}_{2}$. Coronal slices (300 $\left.\mu \mathrm{m}\right)$ containing the $\mathrm{ZI}$ were 495 cut on a Leica VT1200 S vibrating blade microtome (Leica Microsystems Inc., Buffalo

496 Grove, IL, USA) and incubated in a holding chamber with oxygenated artificial cerebral spinal fluid (ACSF) containing (in mM): $125 \mathrm{NaCl}, 2.5 \mathrm{KCl}, 1.25 \mathrm{NaH}_{2} \mathrm{PO}_{4}, 25 \mathrm{NaHCO}_{3}$,

C for 30 minutes, then moved to room temperature for at least an additional 20 minutes before transfer to the recording chamber.

To validate the effects of CNO on hM4Di-transduced cells in the ZI, current-clamp recordings using potassium methylsulfate-based internal solution (in mM: $120 \mathrm{KMeSO}_{4}$, $20 \mathrm{KCl}, 10$ HEPES, 0.2 EGTA, $8 \mathrm{NaCl}, 4$ Mg-ATP, 0.3 Tris-GTP, 14 Phosphocreatine, pH 7.3 with $\mathrm{KOH}$ ( $\sim 300$ mosmol-1) were used to assess changes in excitability. Whole-cell current-clamp recordings were obtained at $33 \pm 1^{\circ} \mathrm{C}$ from $\mathrm{ZI}$ cells expressing mCherry. A recording chamber heater and an in-line solution heater (Warner Instruments) were used to control and monitor the bath temperature throughout the experiment. Cells were 508 visually identified using an upright microscope (Nikon Eclipse FN1) equipped with 509 differential interference contrast optics with infrared illumination and fluorescent 510 microscopy. Spontaneously active cells were injected with hyperpolarizing current (-10 to $511-50 \mathrm{pA}$ ) to bring their membrane potentials to between -80 and $-70 \mathrm{mV}$. A $500 \mathrm{~ms}$ 512 depolarizing current (10 to $120 \mathrm{pA}$ ) was injected to elicit between 2 and 5 action potentials.

513 The current injection repeated every 15 seconds until the cell fired stably and consistently.

514 Following this stabilization, 10 additional recordings were acquired before bath application 515 of either $10 \mu \mathrm{M} \mathrm{CNO}$ or vehicle in ACSF. Recordings of the same current injection were 516 continued every 15 seconds for approximately 5 minutes, until the cell fired consistently 
517 and stably. Ten additional recordings were performed. The number of action potentials elicited during each depolarizing current injection were used to assess excitability. Values for before and after CNO or vehicle application were averaged across five traces and compared. Current clamp signals were acquired at $100 \mathrm{kHz}$ and filtered at $10 \mathrm{kHz}$.

\section{Optogenetically assisted Circuit Mapping}

For the optogenetically assisted circuit mapping experiments, recordings were done using a cesium gluconate-based internal solution containing (in mM): 120 cesium and 0.5 GTP, pH 7.4 adjusted with $\mathrm{CsOH}$ ( 290 mOsm). Whole-cell voltage-clamp recordings were obtained at $33 \pm 1^{\circ} \mathrm{C}$ from visually identified tdTomato-expressing and non-expressing ZI neurons using differential interference contrast optics with infrared

528 illumination and fluorescence microscopy. Optically evoked inhibitory postsynaptic duration with an interval of $200 \mathrm{~ms}$ between pulses were delivered at $10 \mathrm{~Hz}$ to drive paired synaptic responses. Paired pulse ratios (PPR) were determined by the ratio of the amplitude of the peak evoked by the second pulse divided by the amplitude of the peak evoked by the first pulse. Signals were acquired at $100 \mathrm{kHz}$ and filtered at $10 \mathrm{kHz}$.

536 At the end of each experiment, mice were deeply anesthetized with $1.25 \%$ Avertin 537 anesthesia (2,2,2-tribromoethanol and tert-amyl alcohol in $0.9 \% \mathrm{NaCl} ; 0.025 \mathrm{ml} / \mathrm{g}$ body 538 weight) i.p., then perfused transcardially with $0.9 \% \mathrm{NaCl}\left(37^{\circ} \mathrm{C}\right)$, followed by $100 \mathrm{~mL}$ of 539 ice-cold 4\% paraformaldehyde in phosphate buffer solution (PFA/PB). The brain was 
540 dissected and post fixed in $4 \%$ PFA/PB overnight at $4^{\circ} \mathrm{C}$. After cryoprotection in $30 \%$ 541 sucrose/PB for $48 \mathrm{~h}$, coronal sections $(30-45 \mu \mathrm{m})$ were obtained using a freezing sliding

542 microtome and stored in $0.1 \mathrm{M}$ Phosphate Buffered Saline (PBS), pH 7.4 containing $543 \quad 0.01 \%$ sodium azide (Sigma) at $4^{\circ} \mathrm{C}$ until immunostaining. Sections were rinsed in PBS, 544 incubated in $0.1 \%$ Triton X-100 in PBS for 10 minutes at room temperature and blocked 545 in 5\% normal goat serum (NGS) (Vector Labs, Burlingame, CA) with 0.1\% Triton X-100, $546 \quad 0.05 \%$ Tween-20 and $1 \%$ bovine serum albumin (BSA) for 30 minutes at room 547 temperature. Sections were then incubated for $72 \mathrm{~h}$ at $4^{\circ} \mathrm{C}$ in mouse anti-PKC $\delta(1: 1000$, 548 BD Biosciences, 610397), rabbit anti-Phospho-c-Fos (1:2000, Cell Signaling Technology, 5348) or rat anti-mCherry (1:500, Invitrogen, M11217) in 1.5\% NGS blocking solution with 0.1\% Triton X-100, 0.05\% Tween-20 and 1\% BSA. Sections were then rinsed in PBS and incubated in Alexa Fluor 647-conjugated goat anti-mouse (1:100, Invitrogen, A21235),

552 Alexa Fluor 647-conjugated goat anti-rabbit (1:250, Invitrogen, A21244), or goat anti rat 553 Cy3 (1:250, Invitrogen, A10522) secondary antibodies in 1.5\% NGS blocking solution with 0.1\% Triton $\mathrm{X}-100,0.05 \%$ Tween 20 and 1\% BSA, protected from light, for $2 \mathrm{~h}$ at room temperature. Sections were then rinsed in PBS, mounted on positively charged glass slides, air-dried and coverslips were placed using Fluoromount-G (Southern Biotech). injections (i.p) 2 weeks post virus injection into the Zl. Mice were housed in their home 559 cages for 1 hour prior to transcardial perfusion, brain dissection and tissue processing. 560 For the mapping of axonal terminals from CeA-PKC $\delta$ neurons, PKC $\delta$-cre mice injected 561 with AAV9-Syn-Flex-ChrimsonR-TdTomato or AAV8-hSyn-DIO-mCherry were 562 transcardially perfused at least 4 weeks after the brain injections. For the mCherry- 
563 injected brains, $30 \mu \mathrm{m}$ coronal sections from the entire brain where collected and 564 immunostained for mCherry as described above. No staining was performed for the 565 Chrimson-R-injected brains.

566

567

568

569

570

571

572

573

574

575

576

577

578

579

580

581

Imaging and analysis

For confocal studies, images were acquired using a Nikon A1R laser scanning confocal microscope. 2X (for low magnification), 20X (for high magnification) or 40X (oilimmersion for higher magnification) objectives were used. The experimental conditions for image collection including laser intensity, gain, and pinhole were identical for experiments. Multiple channels (GFP, RFP and CY5) were used for sequential image acquisition where $Z$ stacks data collection was done at $0.9 \mathrm{~mm}$. Following acquisition, images were consolidated using NIS Elements software with automatic stitching of subsequent images, and conversion of stacks into maximum intensity z-projections. Quantitative analysis of CeA and $\mathrm{ZI}$ imaging data was performed between bregma -0.82 and -1.82 and bregma -1.06 and -2.54 , for CeA and ZI, respectively. Anatomical limits of each region were identified based on the mouse brain atlas (Paxinos and Franklin, 2001). Number of positive cells were quantified manually for each channel using NIS Elements software using one section per rostro caudal level for each mouse. Co-labeled cells were identified by NIS Elements software automatically and were further visually corroborated by an experimenter.

For the mapping of axonal terminals from CeA-PKC $\delta$ neurons, coronal slices from the entire brain of PKCס-cre mice injected with AAV9-Syn-Flex-ChrimsonR-TdTomato or AAV8-hSyn-DIO-mCherry, collected and immunostained $120 \mu \mathrm{m}$ apart from each other, were visually inspected for the presence of fluorescent axonal terminals using a $20 \mathrm{X}$ 
586 objective in a Nikon A1R laser scanning confocal microscope. Classic morphological 587 criteria, defined as the presence of varicosities and the thickness and organization pattern 588 of the signal, was used to distinguish labeled terminals (very thin fibers with numerous 589 ramifications and varicosities) from fibers of passage (thicker fibers without ramifications 590 and varicosities) as previously described (Bernard et al.,1993). Low and high 591 magnification images of all the brain sections containing terminals were acquired and the 592 anatomical localization of the terminals was then determined using a Mouse Brain Atlas 593 (Paxinos and Franklin, 2001) Representative images of terminals were collected using a 594 40X oil-immersion objective.

Semi-quantitative analysis of the areas containing axonal terminals was done based on the density of terminals observed and are reported as sparce $(+)$, moderate $597(++)$ and dense $(+++)$ in the defined area. A similar analysis was also performed with the 598 brain of experiment 265945645 of the Mouse Brain Connectivity Atlas of the Allen Brain Institute (http://connectivity.brain-map.org/). This brain was identified using the Source Search tool on the Mouse Brain Connectivity Atlas website and filtering for the CeA as the brain region and Prkcd-GluCla-CFP-IRES-Cre as the mouse line of interest. The

602 tracer injected is described as EGFP; the stereotaxic coordinates for the injection were 603 AP -1.82 mm, ML $-2.65 \mathrm{~mm}$, DV $-4.25 \mathrm{~mm}$ and the injection volume was $0.02 \mathrm{~mm}^{3}$.

Data are presented as mean \pm SEM. Statistical analyses was conducted using 606 GraphPad Prism (v8.0). Unpaired/paired two-tailed t-test, Wilcoxon two-tailed matched 607 pair signed rank test, one-way analyses of variance (ANOVA) followed by Tukey's 608 multiple comparison test and two-way ANOVA followed by Dunnett's multiple comparison 
609 test were used. The significance level was set at $p<0.5$. Sample sizes and $p$ values are 610 indicated in figure legends. Detailed information on each statistical test performed are 611 shown in Supplemental Table 1.

\section{Acknowledgements}

This research was supported by the National Center for Complementary and

Competing Interests: The authors declare no conflict of interests.

\section{References:}

1. Dworkin RH. An overview of neuropathic pain: syndromes, symptoms, signs, and several mechanisms. Clin J Pain. 2002;18(6):343-34910.1097/00002508-200211000-00001.

2. Treede RD, Jensen TS. Campbell, J.N. Cruccu, G. Dostrovsky, J.O. Griffin, J.W. Hansson, P. Hughes, R. Nurmikko, T. Serra, J. Neuropathic pain: redefinition and a grading system for clinical and research purposes.

Neurology.

2008;70(18):1630-

\section{163510.1212/01.wnl.0000282763.29778.59.}

633 3. Bernard JF, Besson, JM. The spino(trigemino)pontoamygdaloid pathway: electrophysiological 634 evidence for an involvement in pain processes. J Neurophysiol. 1990;63(3):473635 49010.1152/jn.1990.63.3.473. 
636

637

638

639

640

641

642

643

644

645

646

647

648

649

650

651

652

653

654

655

656

657

658

659

660

661

662

663

664

665

666

667

668

669

670

671

672

4. Carrasquillo Y, Gereau RW 4th. Activation of the extracellular signal-regulated kinase in the amygdala modulates pain perception. $\mathrm{J}$ Neurosci. 155110.1523/JNEUROSCI.3536-06.2007.

5. Bushnell MC, Ceko M, Low LA. Cognitive and emotional control of pain and its disruption in chronic pain. Nat Rev Neurosci. 2013;14(7):502-51110.1038/nrn3516.

6. Neugebauer V, Li W, Bird GC, Han JS. The amygdala and persistent pain. Neuroscientist. 2004;10(3):221-23410.1177/1073858403261077.

7. Zald $\mathrm{DH}$. The human amygdala and the emotional evaluation of sensory stimuli. Brain Research Reviews 2003;41:88-123.10.1016/s0165-0173(02)00248-5.

8. Wilson TD, Valdivia S, Khan A, Ahn HS, Adke AP, Martinez Gonzalez S, Sugimura YK, Carrasquillo Y. Dual and Opposing Functions of the Central Amygdala in the Modulation of Pain. Cell Rep. 2019;29(2):332-346 e33510.1016/j.celrep.2019.09.011.

9. Ricardo JA. Efferent connections of the subthalamic region in the rat. II. The zona incerta. Brain Research. 1981;214(1):43-6010.1016/0006-8993(81)90437-6.

10. Mitrofanis J. Some certainty for the "zone of uncertainty"? Exploring the function of the zona incerta. Neuroscience. 2005;130(1):1-1510.1016/j.neuroscience.2004.08.017.

11. Zhou M, Liu Z, Melin MD, Ng YH, Xu W, Sudhof TC. A central amygdala to zona incerta projection is required for acquisition and remote recall of conditioned fear memory. Nat Neurosci. 2018;21(11):1515-151910.1038/s41593-018-0248-4.

12. Zhang $X$, Van Den Pol A. Rapid binge-like eating and body weight gain driven by zona incerta GABA neuron activation. Science. 2017;356(6340):853-85910.1126/science.aam7100.

13. Chou XL, Wang X, Zhang ZG, Shen L, Zingg B, Huang J, Zhong W, Mesik L, Zhang LI, Tao HW. Inhibitory gain modulation of defense behaviors by zona incerta. Nat Commun. 2018;9(1):115110.1038/s41467-018-03581-6.

14. Zhao ZD, Chen Z, Xiang X, Hu M, Xie H, Jia X, Cai F, Cui Y, Chen Z, Qian L, Liu J, Shang C, Yang Y, Ni X, Sun W, Hu J, Cao P, Li H, Shen WL. Zona incerta GABAergic neurons integrate prey-related sensory signals and induce an appetitive drive to promote hunting. Nat Neurosci. 2019;22(6):921-93210.1038/s41593-019-0404-5.

15. Masri R, Quiton RL, Lucas JM, Murray PD, Thompson SM, Keller A. Zona incerta: a role in central pain. J Neurophysiol. 2009;102(1):181-19110.1152/jn.00152.2009.

16. Wang $H$, Dong $P$, He C, Feng XY, Huang Y, Yang WW, Gao HJ, Shen XF, Lin S, Cao SX, Lian H, Chen J, Yan M, Li XM. Incerta-thalamic Circuit Controls Nocifensive Behavior via Cannabinoid Type 1 Receptors. Neuron. 2020;107(3):538-551 e53710.1016/j.neuron.2020.04.027.

17. Petronilho A, Reis GM, Dias QM, Fais RS, Prado WA. Antinociceptive effect of stimulating the zona incerta with glutamate in rats. Pharmacol Biochem Behav. 2012;101(3):36036810.1016/j.pbb.2012.01.022. 
673

674

675

676

677

678

679

680

681

682

683

684

685

686

687

688

689

690

691

692

693

694

695

696

697

698

699

700

701

702

703

704

705

706

707

708

709

710
18. Moon HC, Park YS. Reduced GABAergic neuronal activity in zona incerta causes neuropathic pain in a rat sciatic nerve chronic constriction injury model. J Pain Res. 2017;10:1125113410.2147/JPR.S131104.

19. Hu TT, Wang RR, Du Y, Guo F, Wu YX, Wang Y, Wang S, Li XY, Zhang SH, Chen Z. Activation of the Intrinsic Pain Inhibitory Circuit from the Midcingulate Cg2 to Zona Incerta Alleviates Neuropathic Pain. J Neurosci. 2019;39(46):9130-914410.1523/JNEUROSCI.168319.2019.

20. Moon HC, Lee YJ, Cho CB, Park YS. Suppressed GABAergic signaling in the zona incerta causes neuropathic pain in a thoracic hemisection spinal cord injury rat model. Neurosci Lett. 2016;632:55-6110.1016/j.neulet.2016.08.035.

21. Hurst JL, West RS. Taming anxiety in laboratory mice. Nat Methods. 2010;7(10):825$82610.1038 /$ nmeth.1500.

22. Vong L, Ye C, Yang Z, Choi B, Chua S, Jr., Lowell BB. Leptin action on GABAergic neurons prevents obesity and reduces inhibitory tone to POMC neurons. Neuron. 2011;71(1):142-15410.1016/j.neuron.2011.05.028.

23. Krashes MJ, Koda S, Ye C, Rogan SC, Adams AC, Cusher DS, Maratos-Flier E, Roth BL, Lowell BB. Rapid, reversible activation of AgRP neurons drives feeding behavior in mice. J Clin Invest. 2011;121(4):1424-142810.1172/JCI46229.

24. Benbouzid M, Pallage V, Rajalu M, Waltisperger E, Doridot S, Poisbeau P, Freund-Mercier MJ, Barrot M. Sciatic nerve cuffing in mice: a model of sustained neuropathic pain. Eur J Pain. 2008;12(5):591-59910.1016/j.ejpain.2007.10.002.

25. Choi Y, Wook Y, Yoon, Na Heung Sik, Kim Sun Ho, Chung Jin Mo. Behavioral signs of ongoing pain and cold allodynia in a rat model of neuropathic pain. Pain. 1994;59(3):36937610.1016/0304-3959(94)90023-X.

26. Colburn RW, Lubin ML, Stone DJ, Jr, Wang Y, Lawrence D, D'Andrea MR, Brandt MR, Liu Y, Flores CM, Qin N. Attenuated cold sensitivity in TRPM8 null mice. Neuron. 2007;54:379-386. 10.1016/j.neuron.2007.04.017.

27. Hargreaves K, Dubner R, Brown F, Flores C, Joris J. A new and sensitive method for measuring thermal nociception in cutaneous hyperalgesia. Pain. 1988;32:77-8810.1016/03043959(88)90026-7.

28. RANDALL LO, SELITTO JJ. A method for measurement of analgesic activity on inflamed tissue. Arch Int Pharmacodyn Ther. 1957;111(4):409-419,

29. Paxinos G, Franklin, K.B.J. The Mouse Brain in Stereotaxic Coordinates.: Academic Press; 2001.

30. Bernard JF, Alden. M., Besson, J.M. The Organization of the Efferent Projections From the Pontine Parabrachial Area to the Amygdaloid Complex: A Phaseolus vulgaris Leucoagglutinin (PHA-L) Study in the Rat. J Comp Neurol. 1993;329(2):201-22910.1002/cne.903290205. 
711 31. Reardon F, Mitrofanis J. Organisation of the amygdalo-thalamic pathways in rats. Anat 712 Embryol (Berl). 2000;201:75-8410.1007/pl00008229.

713 32. Barbier M, Chometton S, Peterschmitt Y, Fellmann D, Risold PY. Parasubthalamic and 714 calbindin nuclei in the posterior lateral hypothalamus are the major hypothalamic targets for 715 projections from the central and anterior basomedial nuclei of the amygdala. Brain Struct Funct. 716 2017;222(7):2961-299110.1007/s00429-017-1379-1.

717 33. Shinonaga YT, M. Mizuno, N. Direct projections from the central amygdaloid nucleus to the 718 globus pallidus and substantia nigra in the cat. Neuroscience. 1992;51(3)10.1016/0306719 4522(92)90308-O.

720 34.Aggleton PJ. The Amygdala A Functional Analysis Second Edition: Oxford University Press; 721 2nd edition; 200021 December 2000. 712 p,

722 35. Hachisuka J, Koerber HR, Ross SE. Selective-cold output through a distinct subset of lamina 723 I spinoparabrachial neurons. Pain. 2020;161(1):185-19410.1097/j.pain.0000000000001710.

724 36. Padilla-Coreano N, Canetta S, Mikofsky RM, Alway E, Passecker J, Myroshnychenko MV, 725 Garcia-Garcia AL, Warren R, Teboul E, Blackman DR, Morton MP, Hupalo S, Tye KM, Kellendonk 726 C, Kupferschmidt DA, Gordon JA. Hippocampal-Prefrontal Theta Transmission Regulates 727 Avoidance Behavior. Neuron. 2019;104(3):601-610 e60410.1016/j.neuron.2019.08.006. 


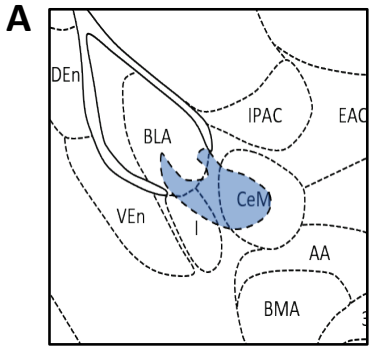

Bregma -0.70

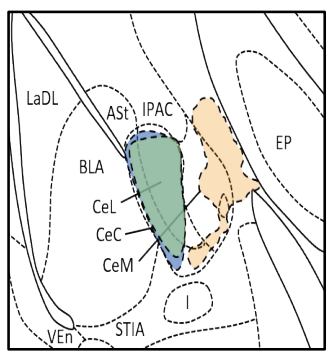

Bregma -1.34

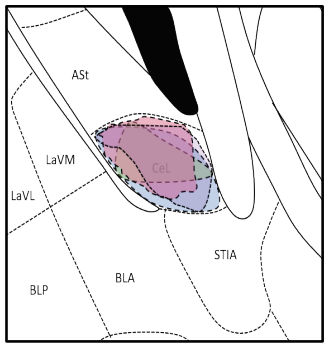

Bregma -1.94

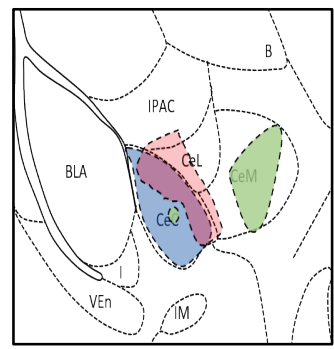

Bregma -0.82

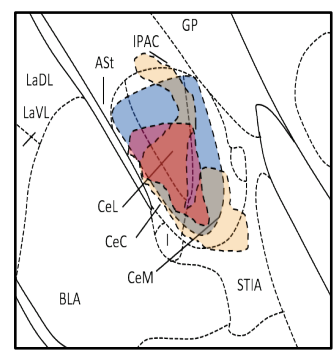

Bregma - 1.46

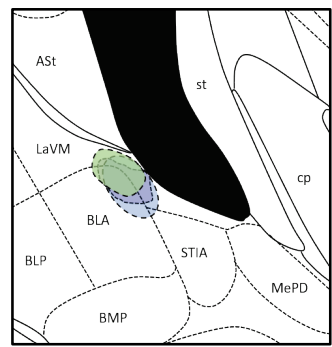

Bregma -2.06

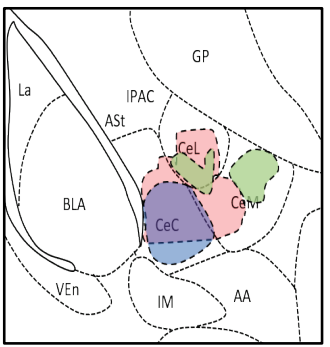

Bregma -0.94

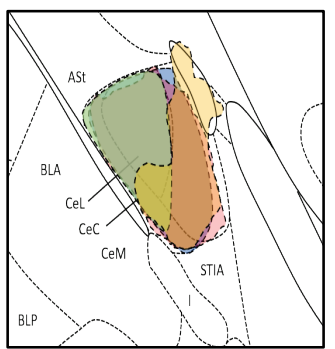

Bregma -1.58

ET 832

ET 835

ET 903

ET 987

265945645

B

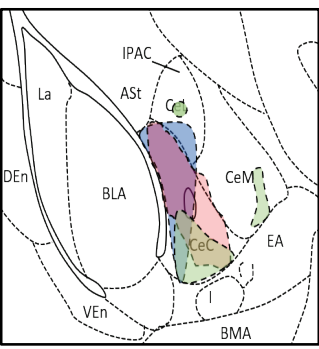

Bregma -1.06

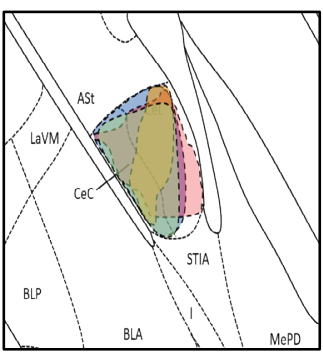

Bregma - 1.70

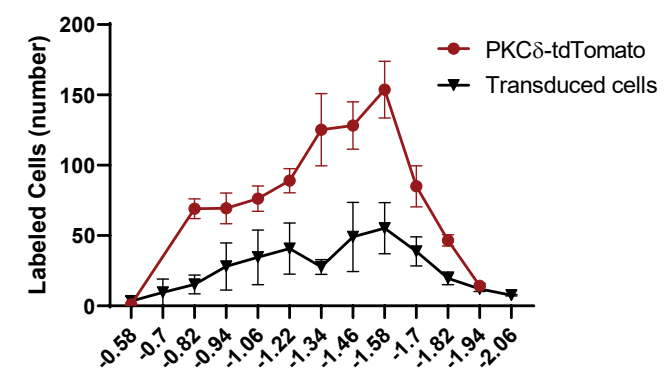

Rostral-Caudal Distribution (relative to bregma)

Bregma -1.22

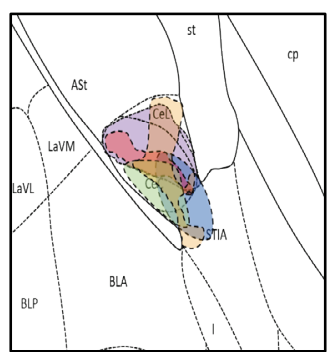

Bregma -1.82

$\rightarrow$ PKC $\delta$-tdTomato

Figure 1 - figure supplement 1. (A) Rostro-caudal distribution of AAV8-hSyn-DIO-mCherry or AAV1-SynFlex-ChrimsonR-tdTomato injection sites in mice used for anatomical analysis. Drawings of injection sites throughout Prkcd-Cre mice brains injected into the CeA with AAV8-hSyn-DIO-mCherry (ET 832 and ET835), AAV1-Syn-Flex-ChrimsonR-tdTomato (ET 903 and ET 987) or EGFP (experiment 265945645 of the Mouse Brain Connectivity Atlas of the Allen Brain Institute- http://connectivity.brain-map.org/). Individual mice are represented in different colors. (B) Mean \pm SEM number of neurons transduced with Chrimson-R-tdTomato or mCherry and PKC $\delta$-tdTomato labeled cells in the CeA as a function of rostrocaudal level relative to bregma ( $n=4$ mice for transduced neurons; $n=5$ mice for PKC $\delta$-tdTomato neurons). 


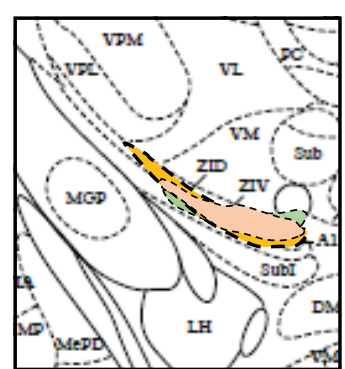

Bregma - 1.46

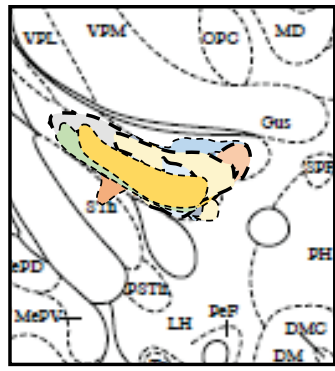

Bregma -2.06

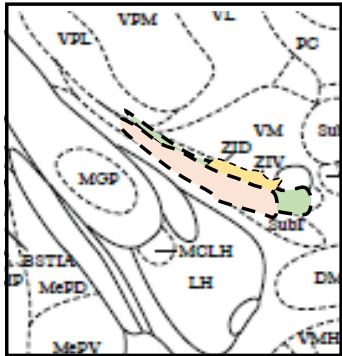

Bregma -1.58

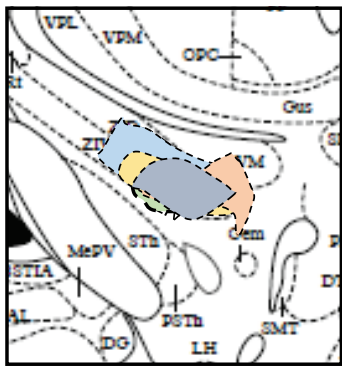

Bregma -2.18

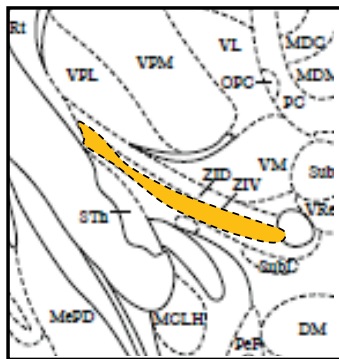

Bregma -1.70

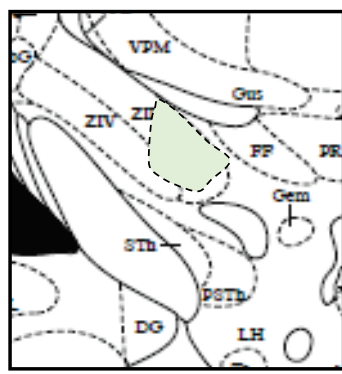

Bregma -2.30

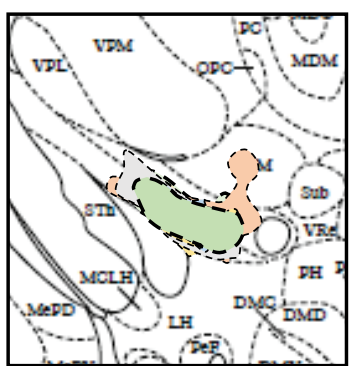

Bregma -1.82

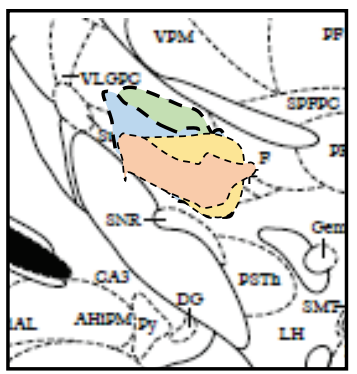

Bregma -2.46

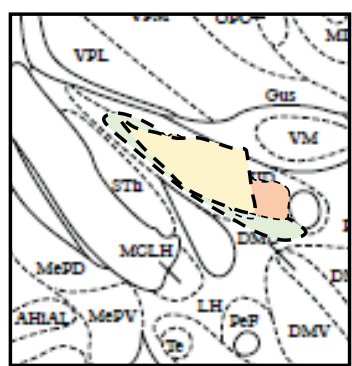

Bregma -1.94

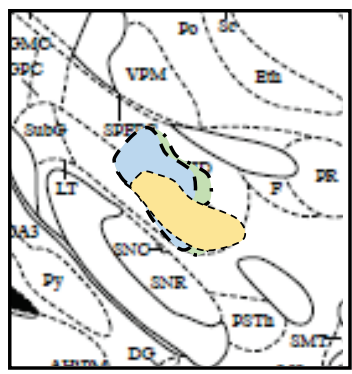

Bregma -2.54

Figure 4 - figure supplement 1. Rostro-caudal distribution AAV-hSyn-DIO-hM4D(Gi)-mCherry injection sites in mice used for behavioral experiments. Drawings of injection sites throughout the VGAT-Cre mice brains injected with AAV-hSyn-DIO-hM4D(Gi)-mCherry into the ZI. Individual mice are represented in different color. 

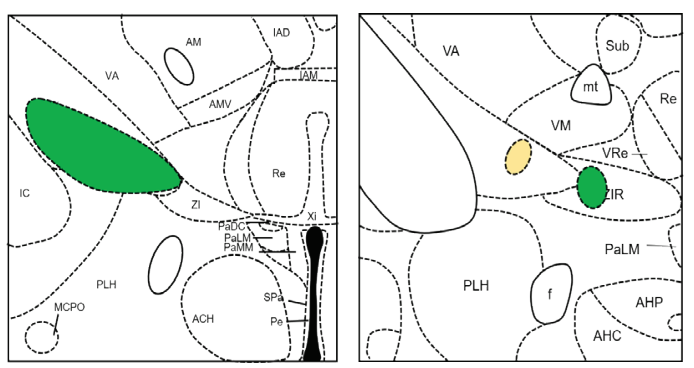

Bregma -0.82

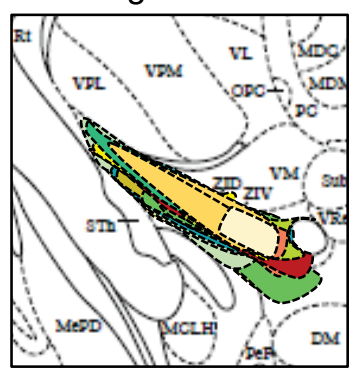

Bregma -1.70

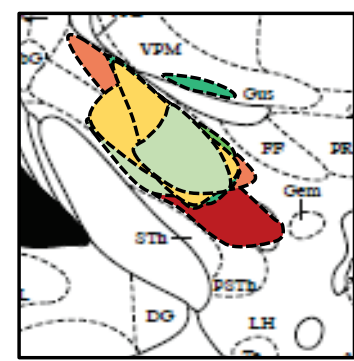

Bregma -2.30

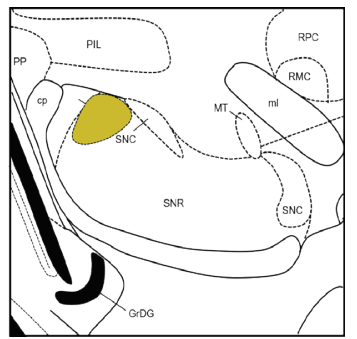

Bregma -3.28

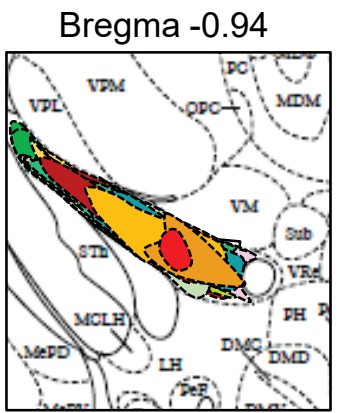

Bregma -1.82

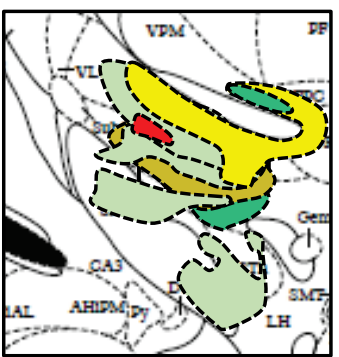

Bregma -2.46

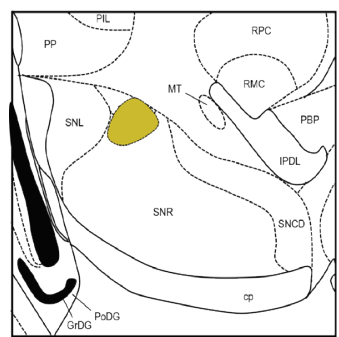

Bregma -3.40

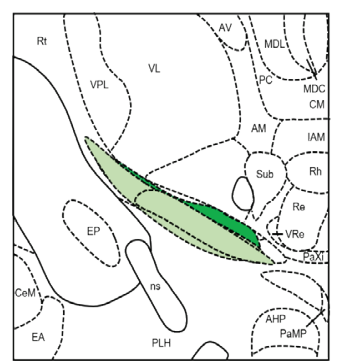

Bregma -1.06

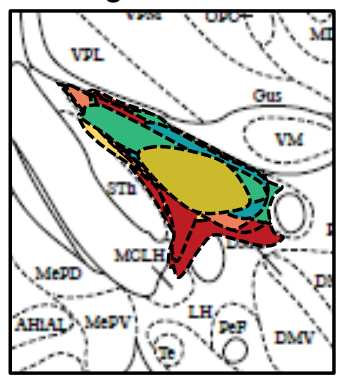

Bregma -1.94

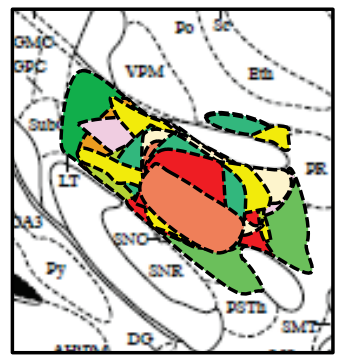

Bregma -2.54

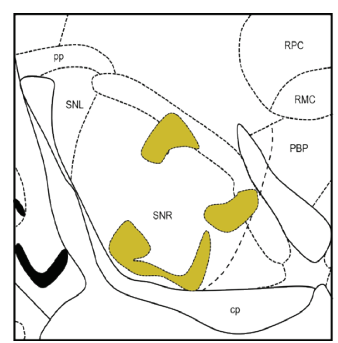

Bregma -3.52

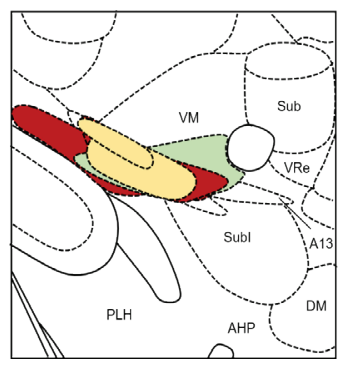

Bregma -1.22

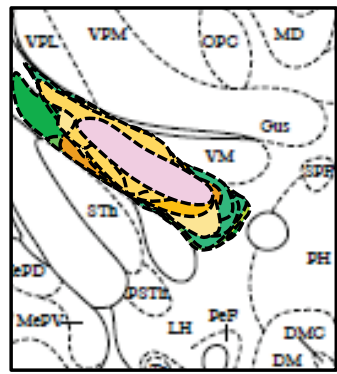

Bregma -2.06

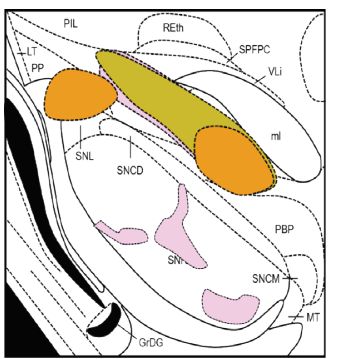

Bregma -3.08

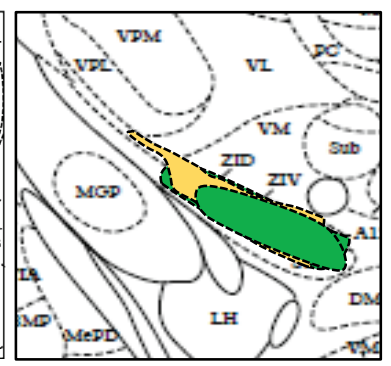

Bregma -1.46

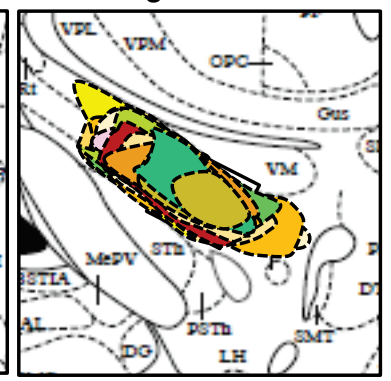

Bregma -2.18

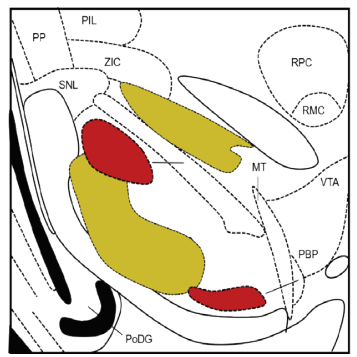

Bregma -3.16

Figure 6 - supplemental figure 1. Rostro-caudal distribution of AAV-hSyn-DIO-hM3D(Gq)-mCherry injection sites in mice used for behavioral experiments. Drawings of injection sites throughout the VGAT-Cre mice brains injected with AAV-hSyn-DIO-hM3D(Gq)-mCherry into the ZI. Individual mice are represented in different color. 


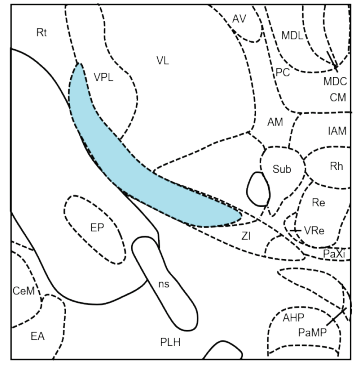

Bregma -1.06

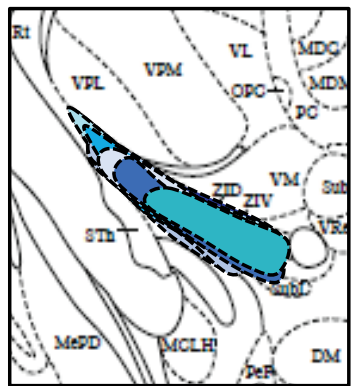

Bregma -1.70

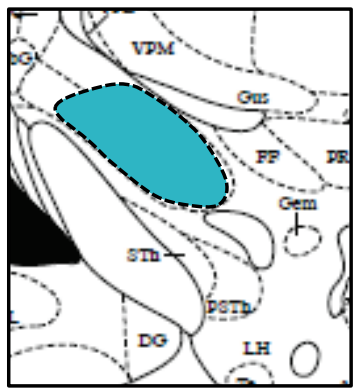

Bregma -2.30

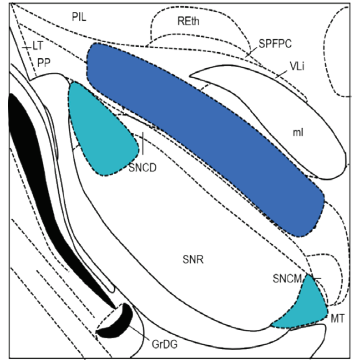

Bregma -3.08

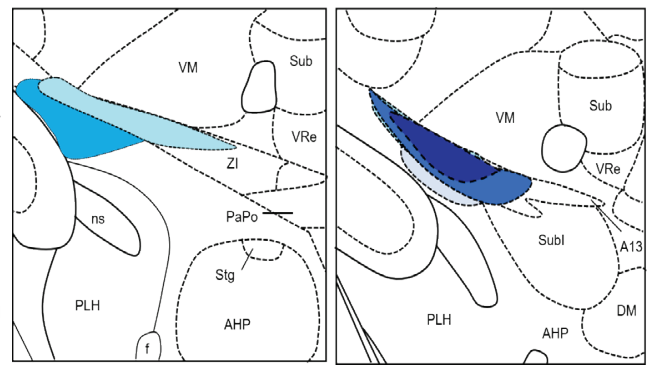

Bregma -1.22

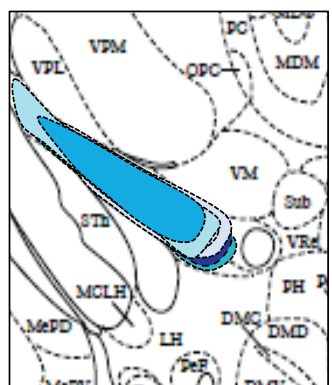

Bregma -1.82

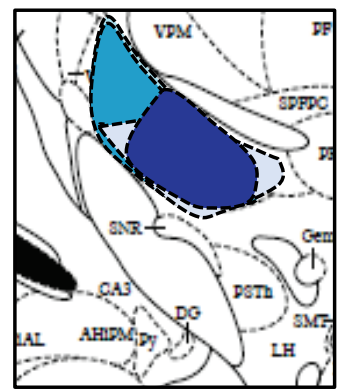

Bregma -2.46

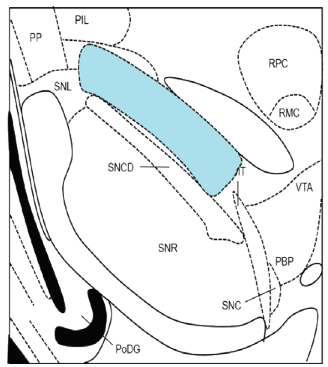

Bregma -3.16

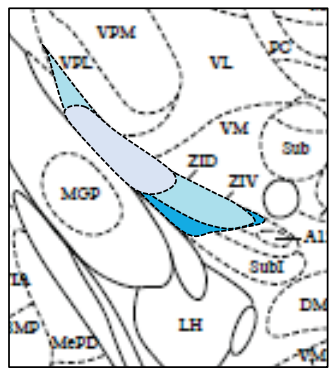

Bregma -1.46

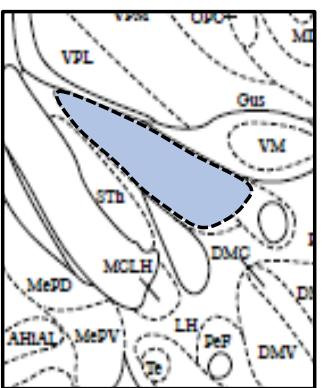

Bregma -1.94

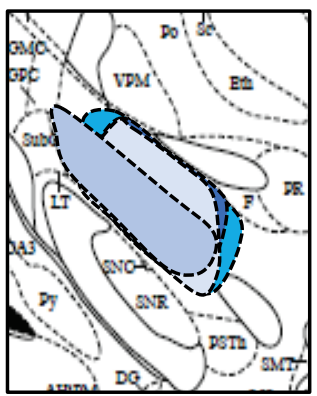

Bregma -2.54

Bregma -2.06

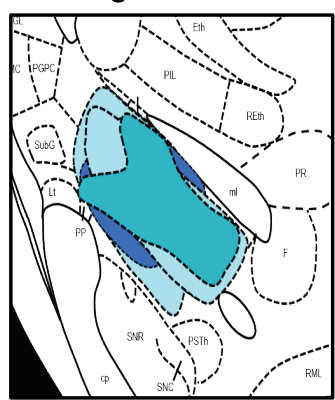

Bregma -2.70

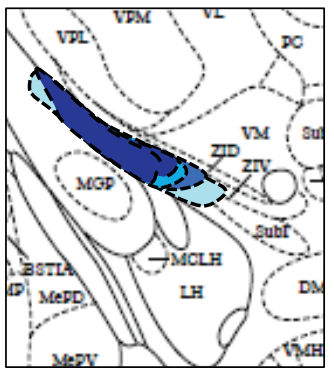

Bregma -1.58

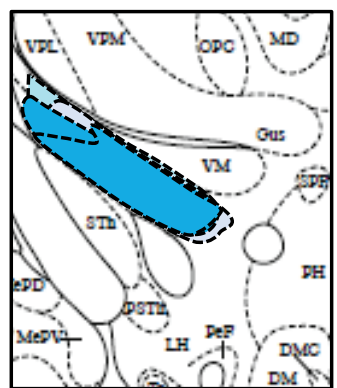

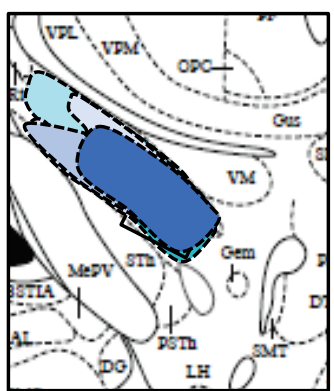

Bregma -2.18

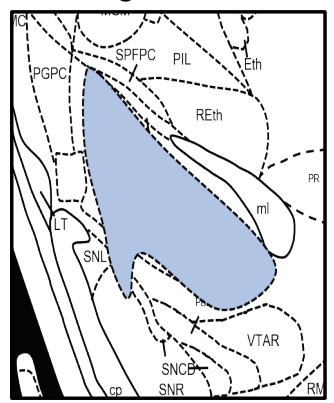

Bregma -2.80

Figure 6 - supplemental figure 2. Rostro-caudal distribution of AAV8-hSyn-DIO-mCherry injection sites in mice used for behavioral experiments. Drawings of injection sites throughout the VGAT-Cre mice brains injected with AAV8-hSyn-DIO-mCherry into the ZI. Individual mice are represented in different color. 
bioRxiv preprint doi: https://doi.org/10.1101/2021.03.10.434888; this version posted March 12, 2021. The copyright holder for this preprint (which was not certified by peer review) is the author/funder, who has granted bioRxiv a license to display the preprint in perpetuity. It is made available under aCC-BY 4.0 International license.

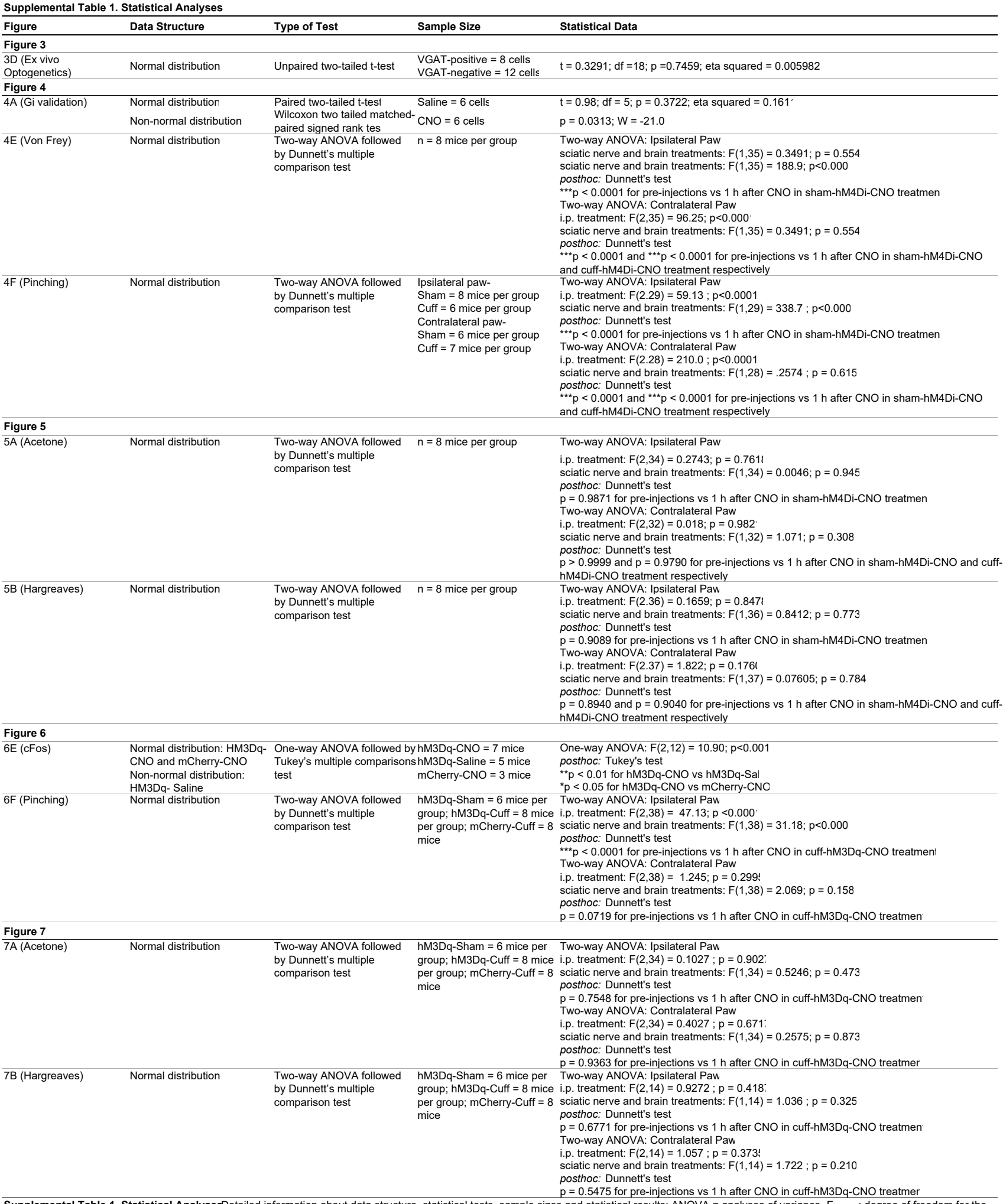

